Article

\title{
Optimization of Multiple Tuned Mass Damper (MTMD) Parameters for a Primary System Reduced to a Single Degree of Freedom (SDOF) Through the Modal Approach
}

\author{
Piotr Wielgos ${ }^{1, *}$ and Robert Geryło ${ }^{2}$ \\ 1 Faculty of Civil Engineering and Architecture, Lublin University of Technology, ul. Nadbystrzycka 40, \\ 20-618 Lublin, Poland; p.wielgos@pollub.pl \\ 2 Building Research Institute, Filtrowa 1, 00-611 Warsaw, Poland; r.gerylo@itb.pl \\ * Correspondence: p.wielgos@pollub.pl
}

\begin{abstract}
The research paper presents a new approach towards constructing motion equations for structures with attached MTMDs (multiple tuned mass dampers). A primary system, with MDOF (multiple dynamic degrees of freedom) was reduced to an equivalent system with a SDOF (single degree of freedom) through the modal approach, and equations from additional MTMDs were added to a thus-created system. Optimization based on $\mathrm{H}_{2}$ and $\mathrm{H}_{\infty}$ for the transfer function associated with the generalized displacement of an SDOF system. The research work utilized GA (genetic algorithms) and SA (simulated annealing method) optimization algorithms to determine the stiffness and damping parameters for individual TMDs. The effect of damping and stiffness (MTMD tuning) distribution depending on the number of TMDs was also analyzed. The paper also reviews the impact of primary system mass change on the efficiency of optimized MTMDs, as well as confirms the results of other authors involving greater MTMD effectiveness relative to a single TMD.
\end{abstract}

Keywords: FEM analysis; vibration control; $\mathrm{H}_{2}$ and $\mathrm{H}_{\infty}$ optimization; MTMD; parameter optimization

\section{Introduction}

The first general formulation of the research problem involving the parameter optimization in a TMD attached to a SDOF structure was suggested by Den Hartog [1]. He determined generally known formulas for TMD tuning and a critical damping ratio in consideration of a TDOF (two degrees of freedom) system with harmonic excitation. Recommendations in terms of the optimal TMD parameter set can also be found in the work by Warburton [2] for a deterministic case, that is, when the main mass of a SDOF system undergoes harmonic excitation. The response of a linear system to broadband response is different to the case of a harmonic excitation, because the first one occurs at system's natural frequencies. In cases where broadband excitation has an almost constant spectrum within the natural frequency range, it is convenient to replace it with white noise. In his work [3] Warburton provided formulas for optimal TMD parameters at exactly such excitation. Similar discussions were conducted by Bakre and Jangid in [4] and [5]. A different approach to the issue of determining TMD parameter values was proposed by Krenk [6], who suggested aligning the ordinates of three points A, B (just like Den Hartog) and one central, between the previous two. He derived a new optimal damping factor value, which is $15 \%$ higher than the classic result.

The most recent work that need mentioning include the one by Batou and Adhikari [7], which focused on an SDOF system with an attached viscoelastic damper. A standard rheological model as a TMD model was used to obtain analytically optimal TMD parameters. Classic results for a TMD with viscous damping can be obtained as a specific case for this damper type. It was also 
demonstrated that using a TMD with viscoelastic damping enables obtaining better vibration absorption compared to an equivalent TMD with viscous damping. Another new approach to a well-known TMD attached to an SDOF system was reviewed in the work by Marian and Giaralis [8]. The authors proposed a TMDI, which combines a classic tuned mass damper (TMD) with a grounded inerter, which counteracts relative acceleration through additional inertia. The work utilized a modified "two point" approach proposed by Den Hartog [1], which enabled determining optimal TMDI parameters. TMD analyses are conducted in various field of science. The study by Peterka et al. [9] involved modelling the vibrations of a drilling mandrel with a vibration damper linked with the mandrel via a viscoelastic coupling. A system with three degrees of freedom with an additionally installed TMD was modelled and the efficiency of such a solution demonstrated.

Multiples TMDs (MTMD) can be introduced to a system in order to improve structural parameters. The efficiency of a spatially distributed MTMD was originally discussed by Bergman et al. [10]. The presented results indicated that structural behavior could be improved when an MTMD is tuned for appropriate structural frequencies, maintaining the total mass ratio at the same level as in the case of a TMD. It was demonstrated that an increased number of damper resulted in the flattening of the response curve over a wider frequency range (Kareem and Kline [11]). The performance of TMD and MTMD was compared and it was shown that the optimal damping values of an MTMD damper were lower than in the case of TMD. The early stages of studies concerning MTMD configurations under simplified and constrained conditions primarily involved in order to reduce the number of associated design variables. For example, in [12], $\mathrm{Xu}$ and Igusa studied MTMD with evenly spaced natural frequencies and an equal constant damping, and based on the asymptotic analysis, they demonstrated that such an MTMD was efficient in reducing primary system response. For a finite number of MTMDs with similar constraints, Joshi and Jangid [13], and Jangid [14] presented optimal MTMD parameters for an undamped and damped primary system, respectively. MTMDs with equal damping factors and evenly spaced natural frequencies were also studied by such authors as Yamaguchi and Harnpornchai [15].

A number of algorithms for selecting optimal MTMD parameter values have been proposed over the years (see Zuo and Nayfeh [16]). The study focused on developing optimization algorithms based on the $\mathrm{H}_{2}$ and $\mathrm{H}_{\infty}$ norms, and aimed at minimizing the response to random and harmonic excitations. $\mathrm{H}_{\infty}$ optimization was also utilized in the work by Zhao [17], for determining optimal ATID (active tuned inerter damper) parameters. The article by Aggumus and Guclu [18] describes a study involving a single-tuned mass damper (STMD) using a model of a building with multiple degrees of freedom (MDOF). It applied an optimization method based on the $\mathrm{H}_{\diamond}$ norm in the STMD control process.

MTMD parameters were studied by Zuo and Nayfeh [19], who utilized $\mathrm{H}_{2}$ optimization. They demonstrated that optimal structures did not have uniformly distributed tuning frequencies or identical damping factors for individual TMDs, and the applied optimization of individual parameters within an MTMD system provided significant improvement of the entire SDOF system with MTMD. In response to the aforementioned study, $\mathrm{Li}$ and $\mathrm{Ni}[20]$ presented an optimization method for MTMDs with non-uniformly distributed mass, based on a gradient with linear search of the objective function. By solving the problem of multi-target optimization based on the objective function in the mode of maximum displacement or frequency response, the authors obtained optimized, unevenly distributed MTMDs. In their work [21], Zuo and Nayfeh applied a method utilizing subgradients of a non-smooth objective function. Besides the gradient methods, also genetic algorithms are used in terms of MTMD optimization, for example, by Ok, Song and Park [22]. Heuristic methods, such as the particle swarm optimization (PSO) were also used. The PSO method for the determination of optimal MTMD parameters was used, among others, in the research by: Leung and Zhang [23], and Zhang et al. [24]. Other methods used for optimizing the objective function in MTMD issues include simulated annealing (SA) applied by Aydin et al. [25] for optimizing the parameters in a multi-story building.

Recent works on MTMD include the one by Kim and Lee [26]. The article discusses the analysis of linear MTMDs with numerous practical configurations, attached to an SDOF structure, subjected to white noise input. Six practical configurations were developed and analyzed comparatively, each 
of which was linearly restricted with distributed tuning factors, mass ratios, damping factors and their combinations. In [27], Stanikzai et al. analyzed TMDs and MTMDs distributed at various degrees of freedom of an MDOF system in a building with base isolation (BI). The analysis involved 40 earthquake ground motions for the adopted pattern of an MDOF structure with attached TMD and MTMDs, and indicated the efficiency of the latter. In [28], Yin et al. analyzed new type of TMD system named pounding tuned mass damper (PTMD). The coupled equations were created by combining the equations of motion of both the bridge and moving vehicles. In order to compare the damping performance, a parametric study of the various numbers and locations, mass ratio and stiffness of the MPTMDs were investigated.

The article presents a new method for developing motion equations for a MTMD structure. The method enables adding single TMDs or MTMD groups to completely different degrees of freedom of the primary system. The system of equations allows for easy MTMD tuning for complex vibrations modes, with MTMDs located in local maxima of these vibrations modes, while still analyzing the SDOF system with attached MTMDs. MTMD parameter optimization based on $\mathrm{H}_{2}$ and $\mathrm{H}_{\infty}$ norms for the transfer function associated with the generalized displacement of an SDOF system was proposed. The research work utilized GA (genetic algorithms) and SA (simulated annealing method) optimization algorithms to determine the stiffness and damping parameters for individual TMDs. The comparison and discussion were based on previously developed models of a primary system with an attached MTMD.

\section{Methods}

\subsection{Original proposal of a motion equations for a primary system with attached MTMDs, reduced to an} SDOF system through the modal approach

In order to be able to model an equivalent system with a single degree of freedom (SDOF) for a system with multiple degrees of freedom using the modal approach, several conditions need to be satisfied, such as the spatial load distribution must be relatively uniform, and in the case of concentrated load, it should be located near the highest ordinate of the first vibration mode, and natural frequencies higher than $\omega_{i}$ are not close to this frequency.

Using this relationship enables obtaining an equation, which describes an equivalent motion of a SDOF system, corresponding to a system with $N$ degrees of freedom. The primary system is reduced through the modal approach. The main assumption is the orthogonality of the main structure $\mathbf{C}$ damping matrix. In general, for $N$ degrees of primary system, the motion equation solution:

$$
\mathbf{M} \ddot{\mathbf{q}}(t)+\mathbf{C} \dot{\mathbf{q}}(t)+\mathbf{K q}(t)=\mathbf{p}(t),
$$

expands into a series of eigenvectors

$$
\mathbf{q}(t)=\sum_{i=1}^{N} \mathbf{a}_{i} \psi_{i}(t)=\mathbf{W} \psi(t),
$$

where $\psi(t)$ is the primary coordinate vector, $\mathbf{a}_{i}$ is $i$-th eigenvector, while $\mathbf{W}$ is the matrix corresponding mode shapes in its columns.

If we include the $N_{i}$ mode of the vibrations, which have the greatest share in the vibration system, the above equation can be expressed as:

$$
\mathbf{q}(t)=\sum_{i=1}^{N_{i}} \mathbf{a}_{i} \psi_{i}(t),
$$

If we normalize the eigenvectors in the manner shown below, we get the following motion equations:

$$
\tilde{m}_{i} \ddot{\psi}_{i}(t)+\tilde{c}_{i} \dot{\psi}_{i}(t)+\tilde{k}_{i} \psi_{i}(t)=\tilde{p}_{i}(t)
$$


where: $i=1,2, \ldots N_{i} \quad \tilde{m}_{i}=\mathbf{a}_{i}^{T} \mathbf{M} \mathbf{a}_{i}=1, \quad \tilde{c}_{i}=\mathbf{a}_{i}^{T} \mathbf{C} \mathbf{a}_{i}=2 \zeta_{i} \omega_{i}, \quad \tilde{k}_{i}=\mathbf{a}_{i}^{T} \mathbf{K} \mathbf{a}_{i}=\omega_{i}^{2}, \quad \tilde{p}_{i}(t)=\mathbf{a}_{i}^{T} \mathbf{p}(t)$.

Designations as in Figure 2. were adopted for the analyzed situation. The diagram shows a beam structure with eliminated degrees of rotation and degrees of freedom along the $X$-axis direction of the global coordinate system.

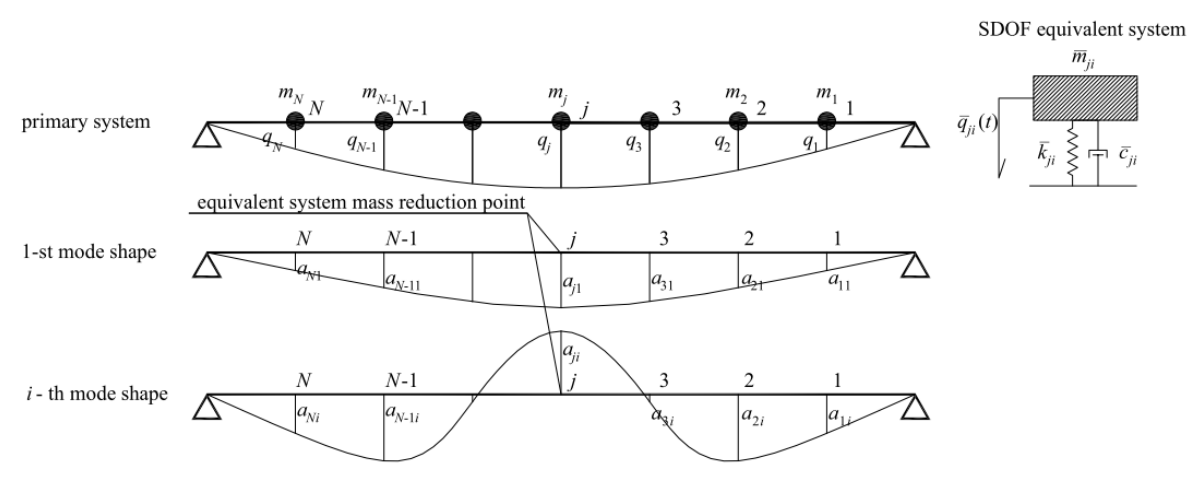

Figure 1. The primary system, adopted first and $i$-th mode shape, together with an SDOF equivalent system.

The following designations for the diagram above were adopted: $j=1,2, \ldots N-$ system degrees of freedom; $i=1,2, \ldots N_{i}$ - number of vibration modes taken into account (further vibration eigenmodes not necessarily included in the analysis) - $i$ index means any subsequent mode number included; $q_{j}(t)$ - displacement of $j$-th degree of freedom;

Assuming that natural frequencies corresponding to the modes, which are decisive for structural vibrations, are separated from each other, the dynamic response in the case of excitations with a selected vibration mode, can be determined using the formula:

$$
q_{j}(t)=\sum_{i=1}^{N_{i}} a_{j i} \psi_{i}(t),
$$

where: $\psi_{i}(t)$ - primary coordinate associated with the $i$-th vibration eigenmode, $a_{j i}$-coordinate of the $i$-th vibration mode of the $j$-th degree of freedom, $N_{i}$ - number of vibration eigenmodes taken into account.

The $S$ point to which the system is reduced, is called the equivalent system mass reduction point. It is adopted at the point of the highest coordinate of the $\mathbf{a}_{i}$ mode shape or the point of application of concentrated load within the system, including, e.g. force originating from a mechanical vibration damper.

After bilaterally dividing the modal motion equation (4) by the coordinate of the $i$-th mode shape of the equivalent system mass reduction point $a_{j i}$ and after normalizing eigenvectors in the form of $\tilde{m}_{i}=\mathbf{a}_{i}^{T} \mathbf{M a} \mathbf{a}_{i}=1$, we get an equivalent system motion equation:

$$
\frac{\tilde{m}_{i}}{a_{j i}^{2}} a_{j i} \ddot{\psi}_{i}(t)+\frac{\tilde{c}_{i}}{a_{j i}^{2}} a_{j i} \dot{\psi}_{i}(t)+\frac{\tilde{k}_{i}}{a_{j i}^{2}} a_{j i} \psi_{i}(t)=\frac{1}{a_{j i}} \tilde{p}_{i}(t),
$$

or

$$
\bar{m}_{j i} \ddot{\bar{q}}_{j i}(t)+\bar{c}_{j i} \dot{\bar{q}}_{j i}(t)+\bar{k}_{j i} \bar{q}_{j i}(t)=\bar{p}_{j i}(t),
$$

where: $i=1,2, . ., N_{i}, \quad \bar{q}_{j i}(t)=a_{j i} \psi_{i}(t)$ - equivalent system displacement, $\quad \bar{m}_{j i}=\tilde{m}_{i} / a_{j i}{ }^{2}=1 / a_{j i}{ }^{2}-$ equivalent mass, $\bar{c}_{j i}=\tilde{c}_{i} / a_{j i}{ }^{2}=2 \zeta_{i} \omega_{i} / a_{j i}{ }^{2}$ - equivalent damping of system, $\bar{k}_{j i}=\tilde{k}_{i} / a_{j i}{ }^{2}=\omega_{i}{ }^{2} / a_{j i}{ }^{2}-$ equivalent stiffness of system, $\bar{p}_{j i}(t)=\tilde{p}_{i}(t) / a_{j i}$ - equivalent force of system. 
A new tuned mass damper (TMD) or multiple TMDs (MTMDs) can be attached to the new system. In general, attached TMDs do not have to be located at the $S$ system reduction point. If the $S$ system reduction point is an attachment point for a single TMD, we get a 2DOF system, which is a well-known issue addressed by Den Hartog in [1], in the case of no damping of the primary system. Of course, TMD is tuned to a frequency near $\omega_{i}$, for which the equivalent system was determined. The case of using MTMDs located in various degrees of freedom of the primary system requires a separate discussion. MTMD arrangement diagram is shown in Figure 2. Of course, each TMD shall be tuned to near frequency $\omega_{i}$.

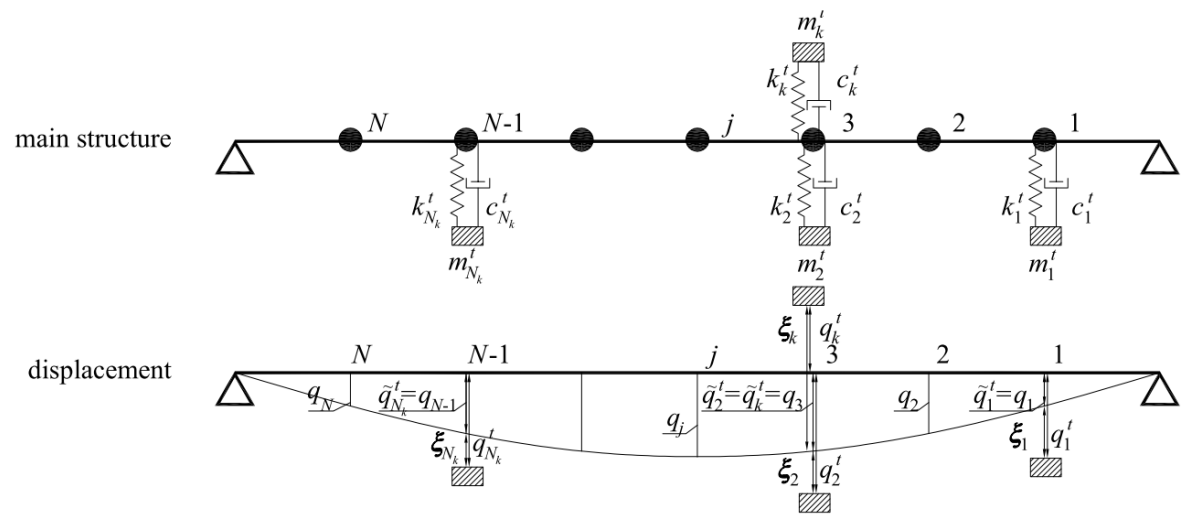

Figure 2. General arrangement diagram for TMDs within the main structure

The following designations for the above diagram were adopted: $k=1,2, \ldots N_{k}-$ TMD degrees of freedom; $m_{k}^{t}, c_{k}^{t}, k_{k}^{t}$ - mass, damping and stiffness of the $k$-th damper; $\xi_{k}(t), q_{k}^{t}(t)$ - relative and absolute displacement of the $k$-th TMD. If we add an $N_{k}$ number of tuned mass dampers to the created system, the new system will have $N_{k}+1$ degrees of freedom (each TMD is an additional degree of freedom).

If we introduce location vectors for each MTMD, with the value 1 present on the degree of freedom to which the tuned mass damper is attached to, we can write the following structural displacement equation, for a degree of freedom to which the k-th TMD is attached to:

$$
\tilde{q}_{k}^{t}(t)=\mathbf{e}_{k}^{T} \mathbf{a}_{i} \psi_{i}(t)=\tilde{a}_{k i} \psi_{i}(t),
$$

where $\mathbf{e}_{k}^{\mathrm{T}}=[0,0,1, \ldots, 0]$ is the location vector of the $k$-th damper. The value 1 is present on the degree of freedom to which the $k$-th TMD is attached to, $\tilde{a}_{k i}=\mathbf{e}_{k}{ }^{T} \mathbf{a}_{i}$ is the ordinate of the $i-$ th eigenvector at the TMD attachment point.

We also have to introduce the value of the displacements, velocity and relative acceleration, which describe the motion of additional MTMDs. Generalized displacements, velocities and relative accelerations for the dampers can be expressed with the following formulas:

$$
\begin{aligned}
& \xi_{k}=q_{k}^{t}-\tilde{q}_{k}^{t}=q_{k}^{t}-\tilde{a}_{k i} \psi_{i}=q_{k}^{t}-\frac{\tilde{a}_{k i}}{a_{j i}} \bar{q}_{j i}, \\
& \dot{\xi}_{k}=\dot{q}_{k}^{t}-\dot{\tilde{q}}_{k}^{t}=\dot{q}_{k}^{t}-\tilde{a}_{k i} \dot{\psi}_{i}=\dot{q}_{k}^{t}-\frac{\tilde{a}_{k i}}{a_{j i}} \dot{\bar{q}}_{j i}, \\
& \ddot{\xi}_{k}=\ddot{q}_{k}^{t}-\ddot{\tilde{q}}_{k}^{t}=\ddot{q}_{k}^{t}-\tilde{a}_{k i} \ddot{\psi}_{i}=\ddot{q}_{k}^{t}-\frac{\tilde{a}_{k i}}{a_{j i}} \ddot{\bar{q}}_{j i},
\end{aligned}
$$

A system of motion equations for such a case, with the number of degrees of freedom of $N_{k}+1$ is shown by the formula below: 


$$
\begin{aligned}
& \bar{m}_{j i} \ddot{\bar{q}}_{j i}(t)+\bar{c}_{j i} \dot{\bar{q}}_{j i}(t)+\bar{k}_{j i} \bar{q}_{j i}(t)=\bar{p}_{j i}(t)+T_{j i} \\
& m_{k}^{t} \ddot{q}_{k}^{t}+c_{k}^{t}\left(\dot{q}_{k}^{t}-\dot{\tilde{q}}_{k}^{t}\right)+k_{k}^{t}\left(q_{k}^{t}-\tilde{q}_{k}^{t}\right)=0
\end{aligned}
$$

The force from each damper, which needs to the attached to the degree of freedom associated with the $k$-th TMD, taking into account absolute values, can be expressed with the following formulas:

$$
\begin{gathered}
T_{k}=-m_{k}^{t} \ddot{q}_{k}^{t}, \\
T_{k}=c_{k}^{t}\left(\dot{q}_{k}^{t}-\dot{\tilde{q}}_{k}^{t}\right)+k_{k}^{t}\left(q_{k}^{t}-\tilde{q}_{k}^{t}\right)=c_{k}^{t}\left(\dot{q}_{k}^{t}-\frac{\tilde{a}_{k i}}{a_{j i}} \dot{\bar{q}}_{j i}\right)+k_{k}^{t}\left(q_{k}^{t}-\frac{\tilde{a}_{k i}}{a_{j i}} \bar{q}_{j i}\right),
\end{gathered}
$$

whereas while using relative values:

$$
\begin{gathered}
T_{k}=-m_{k}^{t}\left(\ddot{\xi}_{k}+\frac{\tilde{a}_{k i}}{a_{j i}} \ddot{\bar{q}}_{j i}\right), \\
T_{k}=c_{k}^{t} \dot{\xi}_{k}+k_{k}^{t} \xi_{k},
\end{gathered}
$$

Changing to generalized force, for absolute values we get:

$$
\begin{gathered}
T_{j i}=\frac{1}{a_{j i}} \sum_{k=1}^{N_{k}} \tilde{a}_{k i} T_{k}=-\sum_{k=1}^{N_{k}} \frac{\tilde{a}_{k i}}{a_{j i}} m_{k}^{t} \ddot{q}_{k}^{t}, \\
T_{j i}=\frac{1}{a_{j i}} \sum_{k=1}^{N_{k}} \tilde{a}_{k i} T_{k}=\sum_{k=1}^{N_{k}} \frac{\tilde{a}_{k i}}{a_{j i}} c_{k}^{t}\left(\dot{q}_{k}^{t}-\frac{\tilde{a}_{k i}}{a_{j i}} \dot{\bar{q}}_{j i}\right)+\sum_{k=1}^{N_{k}} \frac{\tilde{a}_{k i}}{a_{j i}} k_{k}^{t}\left(q_{k}^{t}-\frac{\tilde{a}_{k i}}{a_{j i}} \bar{q}_{j i}\right),
\end{gathered}
$$

and for relative quantities:

$$
\begin{gathered}
T_{j i}=\frac{1}{a_{j i}} \sum_{k=1}^{N_{k}} \tilde{a}_{k i} T_{k}=-\sum_{k=1}^{N_{k}} \frac{\tilde{a}_{k i}}{a_{j i}} m_{k}^{t}\left(\ddot{\xi}_{k}+\frac{\tilde{a}_{k i}}{a_{j i}} \ddot{\bar{q}}_{j i}\right), \\
T_{j i}=\frac{1}{a_{j i}} \sum_{k=1}^{N_{k}} \tilde{a}_{k i} T_{k}=\sum_{k=1}^{N_{k}} \frac{\tilde{a}_{k i}}{a_{j i}} c_{k}^{t} \dot{\xi}_{k}+\sum_{k=1}^{N_{k}} \frac{\tilde{a}_{k i}}{a_{j i}} k_{k}^{t} \xi_{k},
\end{gathered}
$$

By substituting the additional damping force values to motion equations, and by using the relationships for absolute values (17), (18), we get:

$$
\begin{aligned}
& \bar{m}_{j i} \ddot{\bar{q}}_{j i}(t)+\bar{c}_{j i} \dot{\bar{q}}_{j i}(t)+\bar{k}_{j i} \bar{q}_{j i}(t)+\sum_{k=1}^{N_{k}} \frac{\tilde{a}_{k i}{ }^{2}}{a_{j i}{ }^{2}} c_{k}^{t} \dot{\bar{q}}_{j i}-\sum_{k=1}^{N_{k}} \frac{\tilde{a}_{k i}}{a_{j i}} c_{k}^{t} \dot{q}_{k}^{t}+\sum_{k=1}^{N_{k}} \frac{\tilde{a}_{k i}{ }^{2}}{a_{j i}{ }^{2}} k_{k}^{t} \bar{q}_{j i}-\sum_{k=1}^{N_{k}} \frac{\tilde{a}_{k i}}{a_{j i}} k_{k}^{t} q_{k}^{t}=\bar{p}_{j i}, \\
& m_{k}^{t} \ddot{q}_{k}^{t}+c_{k}^{t}\left(\dot{q}_{k}^{t}-\frac{\tilde{a}_{k i}}{a_{j i}} \dot{\bar{q}}_{j i}\right)+k_{k}^{t}\left(q_{k}^{t}-\frac{\tilde{a}_{k i}}{a_{j i}} \bar{q}_{j i}\right)=0
\end{aligned}
$$

Whereas when using relative values (19), (20), we get:

$$
\begin{aligned}
& \bar{m}_{j i} \ddot{\bar{q}}_{j i}(t)+\bar{c}_{j i} \dot{\bar{q}}_{j i}(t)+\bar{k}_{j i} \bar{q}_{j i}(t)+\sum_{k=1}^{N_{k}} \frac{\tilde{a}_{k i}{ }^{2}}{a_{j i}{ }^{2}} m_{k}^{t} \ddot{\bar{q}}_{j i}+\sum_{k=1}^{N_{k}} \frac{\tilde{a}_{k i}}{a_{j i}} m_{k}^{t} \ddot{q}_{k}^{t}=\bar{p}_{j i}, \\
& m_{k}^{t}\left(\ddot{\xi}_{k}+\frac{\tilde{a}_{k i}}{a_{j i}} \ddot{\bar{q}}_{j i}\right)+c_{k}^{t} \dot{\xi}_{k}+k_{k}^{t} \xi_{k}=0
\end{aligned},
$$

Motion equations in matrix form, for the most general case, with absolute values, are shown by the relationship below: 


$$
\overline{\mathbf{M}} \ddot{\overline{\mathbf{q}}}+\overline{\mathbf{C}} \dot{\overline{\mathbf{q}}}+\overline{\mathbf{K}} \overline{\mathbf{q}}=\overline{\mathbf{p}},
$$

where:

$$
\begin{aligned}
& \ddot{\overline{\mathbf{q}}}=\left[\begin{array}{c}
\ddot{\bar{q}}_{j i} \\
\ddot{q}_{1}^{t} \\
\ddot{q}_{2}^{t} \\
\vdots \\
\ddot{q}_{k}^{t} \\
\vdots \\
\ddot{q}_{N_{k}}^{t}
\end{array}\right], \dot{\overline{\mathbf{q}}}=\left[\begin{array}{c}
\dot{\bar{q}}_{j i} \\
\dot{q}_{1}^{t} \\
\dot{q}_{2}^{t} \\
\vdots \\
\dot{q}_{k}^{t} \\
\vdots \\
\dot{q}_{N_{k}}^{t}
\end{array}\right], \overline{\mathbf{q}}=\left[\begin{array}{c}
\bar{q}_{j i} \\
q_{1}^{t} \\
q_{2}^{t} \\
\vdots \\
q_{k}^{t} \\
\vdots \\
q_{N_{k}}^{t}
\end{array}\right], \overline{\mathbf{p}}=\left[\begin{array}{c}
\bar{p}_{j i} \\
0 \\
0 \\
\vdots \\
0 \\
\vdots \\
0
\end{array}\right], \\
& \overline{\mathbf{M}}=\left[\begin{array}{ccccccc}
\bar{m}_{j i} & 0 & 0 & \cdots & 0 & \cdots & 0 \\
0 & m_{1}^{t} & 0 & \cdots & 0 & \cdots & 0 \\
0 & 0 & m_{2}^{t} & \cdots & 0 & \cdots & 0 \\
\vdots & \vdots & \vdots & \ddots & \vdots & \ddots & \vdots \\
0 & 0 & 0 & \cdots & m_{k}^{t} & \cdots & 0 \\
\vdots & \vdots & \vdots & \vdots & \vdots & \ddots & \vdots \\
0 & 0 & 0 & \cdots & 0 & \cdots & m_{N_{k}}^{t}
\end{array}\right] \\
& \overline{\mathbf{C}}=\left[\begin{array}{ccccccc}
\bar{c}_{j i}+\sum_{k=1}^{N_{k}} \frac{\tilde{a}_{k i}{ }^{2}{ }_{j i}{ }^{t}}{a_{k}^{t}} & -\frac{\tilde{a}_{1 i}}{a_{j i}} c_{1}^{t} & -\frac{\tilde{a}_{2 i}}{a_{j i}} c_{2}^{t} & \cdots & -\frac{\tilde{a}_{k i}}{a_{j i}} c_{k}^{t} & \cdots & -\frac{\tilde{a}_{N_{k} i}}{a_{j i}} c_{N_{k}}^{t} \\
-\frac{\tilde{a}_{1 i}}{a_{j i}} c_{1}^{t} & c_{1}^{t} & 0 & \cdots & 0 & \cdots & 0 \\
-\frac{\tilde{a}_{2 i}}{a_{j i}} c_{2}^{t} & 0 & c_{2}^{t} & \cdots & 0 & \cdots & 0 \\
\vdots & \vdots & \vdots & \ddots & \vdots & \ddots & \vdots \\
-\frac{\tilde{a}_{k i}}{a_{j i}} c_{k}^{t} & 0 & 0 & \cdots & c_{k}^{t} & \cdots & 0 \\
\vdots & \vdots & \vdots & \vdots & \vdots & \ddots & \vdots \\
-\frac{\tilde{a}_{N_{k} i}}{a_{j i}} c_{N_{k}}^{t} & 0 & 0 & \cdots & 0 & \cdots & c_{N_{k}}^{t}
\end{array}\right], \\
& \overline{\mathbf{K}}=\left[\begin{array}{ccccccc}
\bar{k}_{j i}+\sum_{k=1}^{N_{k}} \frac{\tilde{a}_{k i}{ }^{2}{ }_{j i}{ }^{2}}{k} & -\frac{\tilde{a}_{1 i}}{a_{j i}} k_{1}^{t} & -\frac{\tilde{a}_{2 i}}{a_{j i}} k_{2}^{t} & \cdots & -\frac{\tilde{a}_{k i}}{a_{j i}} k_{k}^{t} & \cdots & -\frac{\tilde{a}_{N_{k} i}}{a_{j i}} k_{N_{k}}^{t} \\
-\frac{\tilde{a}_{1 i}}{a_{j i}} k_{1}^{t} & k_{1}^{t} & 0 & \cdots & 0 & \cdots & 0 \\
-\frac{\tilde{a}_{2 i}}{a_{j i}} k_{2}^{t} & 0 & k_{2}^{t} & \cdots & 0 & \cdots & 0 \\
\vdots & \vdots & \vdots & \ddots & \vdots & \ddots & \vdots \\
-\frac{\tilde{a}_{k i}}{a_{j i}} k_{k}^{t} & 0 & 0 & \cdots & k_{k}^{t} & \cdots & 0 \\
\vdots & \vdots & \vdots & \vdots & \vdots & \ddots & \vdots \\
-\frac{\tilde{a}_{N_{k} i}}{a_{j i}} k_{N_{k}}^{t} & 0 & 0 & \cdots & 0 & \cdots & k_{N_{k}}^{t}
\end{array}\right],
\end{aligned}
$$


From the generalized displacement and load vector let us separate vector blocks associated with the primary ordinate and actual TMD displacements:

$$
\ddot{\overline{\mathbf{q}}}=\left[\begin{array}{c}
\ddot{\mathbf{q}}_{1} \\
\ddot{\mathbf{q}}_{2}
\end{array}\right], \quad \dot{\overline{\mathbf{q}}}=\left[\begin{array}{c}
\dot{\overline{\mathbf{q}}}_{1} \\
\dot{\mathbf{q}}_{2}
\end{array}\right], \quad \overline{\mathbf{q}}=\left[\begin{array}{c}
\overline{\mathbf{q}}_{1} \\
\overline{\mathbf{q}}_{2}
\end{array}\right], \quad \overline{\mathbf{p}}=\left[\begin{array}{c}
\overline{\mathbf{p}}_{1} \\
\overline{\mathbf{p}}_{2}
\end{array}\right],
$$

where:

$$
\begin{gathered}
\ddot{\overline{\mathbf{q}}}_{1}=\left[\ddot{\bar{q}}_{j i}\right], \dot{\overline{\mathbf{q}}}_{1}=\left[\dot{\bar{q}}_{j i}\right], \overline{\mathbf{q}}=\left[\bar{q}_{j i}\right], \overline{\mathbf{p}}=\left[\bar{p}_{j i}\right], \\
\ddot{\overline{\mathbf{q}}}_{2}=\left[\begin{array}{c}
\ddot{q}_{1}^{t} \\
\ddot{q}_{2}^{t} \\
\vdots \\
\ddot{q}_{k}^{t} \\
\vdots \\
\ddot{q}_{N_{k}}^{t}
\end{array}\right], \dot{\overline{\mathbf{q}}}_{2}=\left[\begin{array}{c}
\dot{q}_{1}^{t} \\
\dot{q}_{2}^{t} \\
\vdots \\
\dot{q}_{k}^{t} \\
\vdots \\
\dot{q}_{N_{k}}^{t}
\end{array}\right], \overline{\mathbf{q}}_{2}=\left[\begin{array}{c}
q_{1}^{t} \\
q_{2}^{t} \\
\vdots \\
q_{k}^{t} \\
\vdots \\
q_{N_{k}}^{t}
\end{array}\right], \overline{\mathbf{p}}_{2}=\left[\begin{array}{c}
0 \\
0 \\
\vdots \\
0 \\
\vdots \\
0
\end{array}\right],
\end{gathered}
$$

Similarly, the blocks associated with the discussed degrees of freedom shall also be separated from the matrix $\overline{\mathbf{M}}, \overline{\mathbf{C}}, \overline{\mathbf{K}}$ :

$$
\overline{\mathbf{M}}=\left[\begin{array}{ll}
\overline{\mathbf{M}}_{11} & \overline{\mathbf{M}}_{12} \\
\overline{\mathbf{M}}_{21} & \overline{\mathbf{M}}_{22}
\end{array}\right], \overline{\mathbf{C}}=\left[\begin{array}{ll}
\overline{\mathbf{C}}_{11} & \overline{\mathbf{C}}_{12} \\
\overline{\mathbf{C}}_{21} & \overline{\mathbf{C}}_{22}
\end{array}\right], \overline{\mathbf{K}}=\left[\begin{array}{ll}
\overline{\mathbf{K}}_{11} & \overline{\mathbf{K}}_{12} \\
\overline{\mathbf{K}}_{21} & \overline{\mathbf{K}}_{22}
\end{array}\right] .
$$

The size of blocks $\overline{\mathbf{M}}_{11}, \overline{\mathbf{C}}_{11}, \overline{\mathbf{K}}_{11}$ is $1 \times 1, \overline{\mathbf{M}}_{12}, \overline{\mathbf{C}}_{12}, \overline{\mathbf{K}}_{12}$ is $1 \times N_{k}, \overline{\mathbf{M}}_{21}, \overline{\mathbf{C}}_{21}, \overline{\mathbf{K}}_{21}$ is $N_{k} \times 1$ and of $\overline{\mathbf{M}}_{22}, \overline{\mathbf{C}}_{22}, \overline{\mathbf{K}}_{22}$ is $N_{k} \times N_{k}$. Below you can find the forms of individual blocks of matrices $\overline{\mathbf{M}}, \overline{\mathbf{C}}, \overline{\mathbf{K}}$.

$$
\begin{aligned}
& \overline{\mathbf{M}}_{11}=\left[\bar{m}_{j i}\right], \overline{\mathbf{M}}_{22}=\left[\begin{array}{cccccc}
m_{1}^{t} & 0 & \cdots & 0 & \cdots & 0 \\
0 & m_{2}^{t} & \cdots & 0 & \cdots & 0 \\
\vdots & \vdots & \ddots & \vdots & \ddots & \vdots \\
0 & 0 & \cdots & m_{k}^{t} & \cdots & 0 \\
\vdots & \vdots & \ddots & \vdots & \ddots & \vdots \\
0 & 0 & \cdots & 0 & \cdots & m_{N_{k}}^{t}
\end{array}\right] \\
& \overline{\mathbf{M}}_{12}=\overline{\mathbf{M}}_{21}^{T}=\left[\begin{array}{llllll}
0 & 0 & \cdots & 0 & \cdots & 0
\end{array}\right], \\
& \overline{\mathbf{C}}_{11}=\left[\bar{c}_{j i}+\sum_{k=1}^{N_{k}} \frac{\tilde{a}_{k i}{ }^{2}}{a_{j i}{ }^{2}} c_{k}^{t}\right], \overline{\mathbf{C}}_{22}=\left[\begin{array}{cccccc}
c_{1}^{t} & 0 & \cdots & 0 & \cdots & 0 \\
0 & c_{2}^{t} & \cdots & 0 & \cdots & 0 \\
\vdots & \vdots & \ddots & \vdots & \ddots & \vdots \\
0 & 0 & \cdots & c_{k}^{t} & \cdots & 0 \\
\vdots & \vdots & \ddots & \vdots & \ddots & \vdots \\
0 & 0 & \cdots & 0 & \cdots & c_{N_{k}}^{t}
\end{array}\right], \\
& \overline{\mathbf{C}}_{12}=\overline{\mathbf{C}}_{21}^{T}=\left[\begin{array}{cccccc}
-\frac{\tilde{a}_{1 i}}{a_{j i}} c_{1}^{t} & -\frac{\tilde{a}_{2 i}}{a_{j i}} c_{2}^{t} & \cdots & -\frac{\tilde{a}_{k i}}{a_{j i}} c_{k}^{t} & \cdots & -\frac{\tilde{a}_{N_{k} i}}{a_{j i}} c_{N_{k}}^{t}
\end{array}\right],
\end{aligned}
$$




$$
\begin{aligned}
& \overline{\mathbf{K}}_{11}=\left[\bar{k}_{j i}+\sum_{k=1}^{N_{k}} \frac{\tilde{a}_{k i}{ }^{2}{ }_{j i}{ }^{2}}{k_{k}^{t}}\right], \overline{\mathbf{K}}_{22}=\left[\begin{array}{cccccc}
k_{1}^{t} & 0 & \cdots & 0 & \cdots & 0 \\
0 & k_{2}^{t} & \cdots & 0 & \cdots & 0 \\
\vdots & \vdots & \ddots & \vdots & \ddots & \vdots \\
0 & 0 & \cdots & k_{k}^{t} & \cdots & 0 \\
\vdots & \vdots & \ddots & \vdots & \ddots & \vdots \\
0 & 0 & \cdots & 0 & \cdots & k_{N_{k}}^{t}
\end{array}\right], \\
& \overline{\mathbf{K}}_{21}=\overline{\mathbf{K}}_{12}^{T}=\left[\begin{array}{cccccc}
-\frac{\tilde{a}_{1 i}}{a_{j i}} k_{1}^{t} & -\frac{\tilde{a}_{2 i}}{a_{j i}} k_{2}^{t} & \cdots & -\frac{\tilde{a}_{k i}}{a_{j i}} k_{k}^{t} & \cdots & -\frac{\tilde{a}_{N_{k} i}}{a_{j i}} k_{N_{k}}^{t}
\end{array}\right],
\end{aligned}
$$

The new system suggested by the authors, significantly limits the time consumption of the calculations, and also enables the optimization of MTMD parameters discussed below. In general, $N_{i}$ equivalent systems can be created (as many as natural frequencies are taken into account). Each of these systems, with the number $i$, is a system with $N_{k}+1$ degrees of freedom. Achieving full displacement at the $S$ reduction point, requires determining the response for each of the created systems, and then applying the formula:

$$
\bar{q}_{j}(t)=\sum_{i=1}^{N_{i}} \bar{q}_{j i}(t),
$$

This approach is obviously not convenient. However, the method of reducing the system to an SDOF is very useful for structures with simple static diagrams (beams, cantilever structures such as chimneys, masts), when the lowest vibration natural frequency is taken into account. Next, let us determine the parameters of an SDOF equivalent system. For such a system, using the aforementioned equations, we can create new motion equations with attached single TMDs or MTMDs tuned to a distinguished natural frequency.

\subsection{Determination of a transfer matrix used to determine the objective function in optimization issues}

For a model described with the general motion equation, which is a system with $N$ degrees of freedom, the transfer matrix $\mathbf{H}(\lambda)$, the single element of which $H_{i k}(\lambda)$ is called a transfer function, is a Fourier transform of the impulse response $h_{i k}(t)$. The below equations (39) and (40)show a pair of Fourier transforms, which can be found in the work by Bendat [29]:

$$
\begin{gathered}
H_{i k}(\lambda)=\int_{-\infty}^{\infty} h_{i k}(t) e^{-j \lambda t} d t, \\
h_{i k}(t)=\frac{1}{2 \pi} \int_{-\infty}^{\infty} H_{i k}(\lambda) e^{j \lambda t} d \lambda,
\end{gathered}
$$

where: impulse response $h_{i k}(t)$ is the $i$-th response of the system to the $k$-th excitation in the form of a unit impulse function $\delta_{k}$ (Dirac delta) applied at the initial moment $t=0, j$ means an imaginary unit, $(\lambda)$ means the frequency domain. Therefore, in order to determine impulse responses in the $i$ points of the system, the excitation shall be adopted in the following form:

$$
\mathbf{p}(t)=\left[0,0, \ldots, 0, \delta_{k}(t), 0, \ldots, 0\right]^{T},
$$

and by substituting the aforementioned load vector mode to the equation (1), and after applying the Fourier transform, we get:

$$
\left(\mathbf{K}-\lambda^{2} \mathbf{M}+j \lambda \mathbf{C}\right) \mathbf{H}(\lambda)=\mathbf{I} .
$$

In general, $H_{i k}(\lambda)$ is the complex quantity and can be presented as: 


$$
\begin{gathered}
H_{i k}=\left|H_{i k}\right| e^{j \Theta_{i k}}=\operatorname{Re} H_{i k}+j \operatorname{Im} H_{i k}, \\
\left|H_{i k}\right|=\sqrt{\left(\operatorname{Re} H_{i k}\right)^{2}+\left(\operatorname{Im} H_{i k}\right)^{2}}, \arg H_{i k}=\Theta_{i k}=\operatorname{arctg} \frac{\operatorname{Re} H_{i k}}{\operatorname{Im} H_{i k}},
\end{gathered}
$$

where: $\left|H_{i k}\right|, \Theta_{i k}$ - transfer function module and argument, respectively.

If we assume the denotation of the dynamic stiffness matrix in the form:

$$
\mathbf{G}(\lambda)=\mathbf{K}-\lambda^{2} \mathbf{M}+j \lambda \mathbf{C}=\operatorname{Re} \mathbf{G}+j \operatorname{Im} \mathbf{G},
$$

we get a complex equation:

$$
\operatorname{Re} \mathbf{G} \operatorname{Re} \mathbf{H}-\operatorname{Im} \mathbf{G} \operatorname{Im} \mathbf{H}+j[\operatorname{Im} \mathbf{G} \operatorname{Re} \mathbf{H}+\operatorname{Re} \mathbf{G} \operatorname{Im} \mathbf{H}]=\mathbf{I},
$$

and after breaking down, two real equations:

$$
\begin{aligned}
& \operatorname{Re} \mathbf{G e} \mathbf{H}-\operatorname{Im} \mathbf{G} \operatorname{Im} \mathbf{H}=\mathbf{I}, \\
& \operatorname{Im} \mathbf{G R e} \mathbf{H}+\operatorname{Re} \mathbf{G} \operatorname{Im} \mathbf{H}=\mathbf{0} .
\end{aligned}
$$

Ultimately, we get a matrix of the real and imaginary section of the full transfer matrix $\mathbf{H}(\lambda)$ :

$$
\begin{gathered}
\operatorname{Re} \mathbf{H}=\left\{\operatorname{Re} \mathbf{G}+\operatorname{Im} \mathbf{G}[\operatorname{Re} \mathbf{G}]^{-1} \operatorname{Im} \mathbf{G}\right\}^{-1} \mathbf{I}, \\
\operatorname{Im} \mathbf{H}=[\operatorname{Re} \mathbf{G}]^{-1} \operatorname{Im} \mathbf{G} \operatorname{Re} \mathbf{H},
\end{gathered}
$$

Of course, with an appropriate computational procedure at our disposal, we can directly determine a complex transfer matrix from the formula:

$$
\mathbf{H}(\lambda)=(\mathbf{G}(\lambda))^{-1}
$$

Knowing the transfer matrix, it is possible to determine the structural response within the frequency domain, in the form of:

$$
\mathbf{q}(\lambda)=\mathbf{H}(\lambda) \mathbf{p}(\lambda)
$$

where $q(\lambda), \mathbf{p}(\lambda)$ are the Fournier transforms of the displacement and load vector.

The structure's load Fourier transform is expressed by the formula:

$$
\mathbf{p}(\lambda)=\int_{-\infty}^{\infty} \mathbf{p}(t) e^{-j \lambda t} d t
$$

If we determine the Fourier retransform of the structure's load vector, we get the following relationship:

$$
\mathbf{q}(t)=\frac{1}{2 \pi} \int_{-\infty}^{\infty} \mathbf{H}(\lambda)\left(\int_{-\infty}^{\infty} \mathbf{p}(t) e^{-j \lambda t} d t\right) e^{j \lambda \tau} d \lambda
$$

Therefore, the transfer function module $\left|H_{i k}(\lambda)\right|$ can be used as an objective function in terms of optimization issues. The form of the objective function proposed for the optimization issue will have the following form:

$$
\min J 1(\lambda)=\int_{\omega_{i}-\lambda_{i}}^{\omega_{i}+\lambda_{i}}\left|H_{j j}(\lambda)\right| d \lambda,
$$

where $\omega_{i}$ is the fundamental frequency of the system to which MTMDs were tuned, while $\lambda_{i}$ is the range in which the area under the diagonal transfer graph function is calculated $\left|H_{j j}(\lambda)\right|$. This 
formula takes only the model of the diagonal element $\left|H_{j j}(\lambda)\right|$ in the transfer matrix $\mathbf{H}(\lambda)$. This corresponds to strengthening the response after passing a system at the $j$ degree of freedom after an excitation at the same $j$ degree of freedom. The transfer function argument, which is the phase shift angle between the load and the response, is omitted in objective functions, since it does not determine the MTMD operational effectiveness. This proposal is very similar to the objective functions known in the source literature as the $\mathrm{H}_{2}$ norm, which correspond to the objective functions based on the spectral response of a structure to white noise input.

An objective function form similar to the $\mathrm{H}_{\infty}$ norm known from the source literature was also proposed. In this case, the optimization criterion is the minimization of the maximum values of the transfer function $\left|H_{j j}(\lambda)\right|$ (see Figure 3), which can be described by the formula:

$$
\min J 2(\lambda)=\max \left|H_{j j}(\lambda)\right| \quad \lambda \in\left\langle\omega_{i}-\lambda_{i} ; \omega_{i}+\lambda_{i}\right\rangle
$$

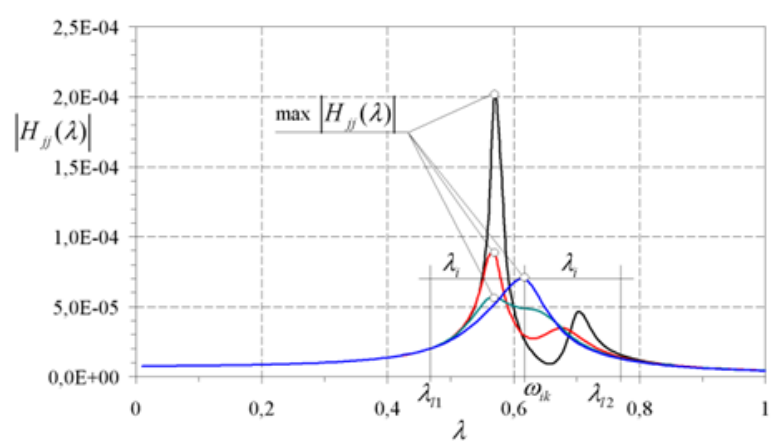

Figure 3. Objective function determination diagram (maximum value within the range)

Both cases of the transfer function form and optimization included the transfer function of the equivalent system $\left|\bar{H}_{j j}(\lambda)\right|$, associated with the $j$ degree of freedom, which was adopted as the equivalent system reduction point, and with the $i$-th mode of structural natural vibrations and the corresponding value of generalized displacement, associated with the primary ordinate $\bar{q}_{j i}(t)=a_{j i} \psi_{i}(t)$.

\subsection{The issue of optimization}

The analysis used two optimization methods in the form of a standard genetic algorithm (GA), and a simulated annealing (SA) algorithm. Ready libraries for the DELPHI software environment were used.

The procedure for the numerical determination of the optimal parameters can be summed up in the following steps:

1. Calculations of the eigenvalues and eigenvectors in FEM software, adopting baseline frequency $\omega_{i}$, to which MTMDs will be tuned;

2. Adopting a point for reducing a system to an equivalent SDOF system, normalization of eigenvectors and determination of the parameters for the equation (7);

3. Adopting a TMD number, and initial parameters $m_{k}^{t}, c_{k}^{t}, k_{k}^{t}$ for the TMDs;

4. Adopting variables, which will be subject to the optimization process, e.g. $c_{k}^{t}, k_{k}^{t}$ for a constant value of $m_{k}^{t}$ (different constant parameter options possible);

5. Selection of the optimization method (GA or SA) and the optimization issue, calculations of the equivalent system transfer function $\left|\bar{H}_{j j}(\lambda)\right|$ and the determination of values using the (55) or (56) at each optimization step.

6. After reaching the desired calculation accuracy (change of the $J 1$ or $J 2$ ) value, saving the obtained MTMD parameters and calculations of the equivalent system transfer function 
$\left|\bar{H}_{i j}(\lambda)\right|$ associated with the $j$ degree of freedom, which was adopted as the equivalent system reduction point.

\section{Results}

\subsection{Input data}

The analysis adopted a cantilever structure in the form of a chimney made of S235 steel, with a height of $h=160 \mathrm{~m}$. The chimney parameters were selected so as to obtain the first circular natural frequency equal to $\omega_{1}=1 \mathrm{rad} / \mathrm{s}$. The chimney diameter and its wall thickness were adopted as a constant, along its entire height. These values equal to, respectively: $D=4010 \mathrm{~mm}, g=25.5 \mathrm{~mm}$. The cantilever structure was divided into 40 beam elements with the following parameters: Young modulus $E=210 \mathrm{GPa}$, Poisson coefficient $v=0.3$ and density $\rho=7850 \mathrm{~kg} / \mathrm{m}^{3}$. First 3 natural vibration modes, and their corresponding eigenvalues were calculated. FEA software was used to import a normalized eigenvector for the first mode of the natural vibrations, which was then used to construct motion equations for the structure reduced to SDOF system with attached TMD.

Figure 4 shows a FEM model with the numberings of the bars and modes, and a presentation of the first three mode shapes and normalized eigenvectors.

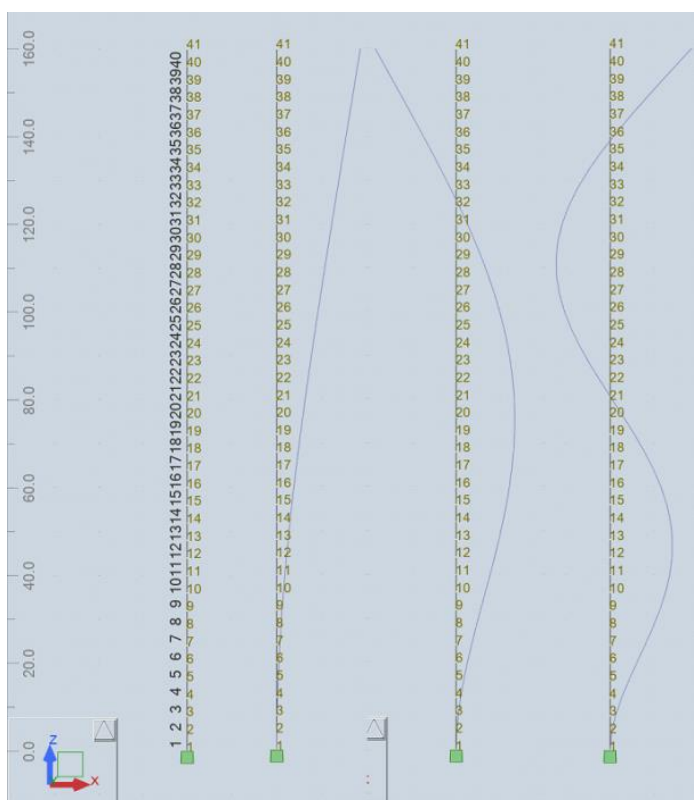

(a)

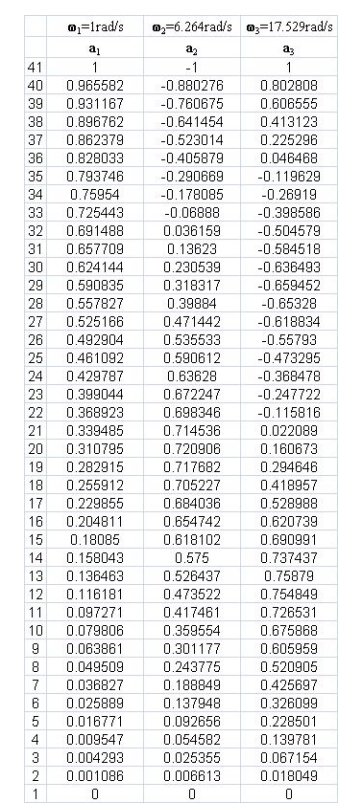

(b)

Figure 4. (a) FEM model of the chimney and the first three mode shapes; (b) normalized eigenvectors for the first three eigenvalues.

The analysis adopted the issue of tuning TMD and MTMDs to the first natural frequency $\omega_{1}=1 \mathrm{rad} / \mathrm{s}$. For this frequency, the adopted structural damping was in the form of a damping ratio $\zeta_{1}=0.02$. The chimney tip (node 41 ) was selected as the $S$ point for reducing the structure to an SDOF equivalent system. The problem involved finding optimal parameters for a single TMD and MTMDs in the form of 2, 4, 8, and 20 TMDs located also at the place of the highest ordinate of the first vibration mode, which was node 41 . New $\overline{\mathbf{M}}, \overline{\mathbf{C}}, \overline{\mathbf{K}}$ matrices for the equivalent system were determined based on the presented modal reduction equations. The equivalent mass determined from the formula $\bar{m}_{j i}=\tilde{m}_{i} / a_{j i}{ }^{2}=1 / a_{j i}{ }^{2}$ (where $j$ is the system point of reduction, namely node 41, whereas $i=1$ corresponds to the first natural vibration mode) amounted to $\bar{m}_{41,1}=\tilde{m}_{1} / a_{41,1}{ }^{2}=10^{5} \mathrm{~kg}$, equivalent stiffness was $\bar{k}_{41,1}=\tilde{k}_{1} / a_{41,1}{ }^{2}=\omega_{1}^{2} / a_{41,1}{ }^{2}=10^{5} \mathrm{~kg} / \mathrm{s}^{2}=100 \mathrm{kN} / \mathrm{m}$ and equivalent damping 
$\bar{c}_{41,1}=\tilde{c}_{1} / a_{41,1}{ }^{2}=2 \zeta_{1} \omega_{1} / a_{41,1}{ }^{2}=4 \cdot 10^{3} \mathrm{~kg} / \mathrm{s}$. Numerical optimization was conducted for all cases. For each case, the total MTMD mass was equal to the mass of the single TMD, which was adopted as equal to $m_{1}^{t}=2 \cdot 10^{3} \mathrm{~kg}$, therefore, the mass factor was $\mu=m_{1}^{t} / \bar{m}_{41,1}=0.02$. For all other cases, TMD masses were determined from the formula $m_{k}^{t}=\mu \cdot \bar{m}_{41,1} / N_{k}$. The natural circular frequency for a single TMD was determined from the formula $\omega_{k}^{t}=\sqrt{k_{k}^{t} / m_{k}^{t}}$, whereas the damping factor values for individual TMDs were determined from the formula $c_{k}^{t}=2 \zeta_{k}^{t} m_{k}^{t} \omega_{k}$. Stiffness $k_{k}^{t}$ was used to determine the dimensionless tuning ratio for a single TMD $\beta_{k}=\omega_{k}^{t} / \omega_{1}$. Initial tuning adopted for all dampers within the optimization amounted to $\beta_{k}=1$. This constituted a base to adopt the starting values $k_{k}{ }^{t}$. Table 1 shows the adopted constant masses of a single TMD, and the adopted starting values of the $k_{k}^{t}$ and $\zeta_{k}^{t}$ MTMD parameters (values subject to optimization), depending on the TMD number.

Table 1. Mass values for individual TMDs, and $k_{k}^{t}$ and $\zeta_{k}^{t}$ starting values.

\begin{tabular}{cccc}
\hline TMD number & $\begin{array}{c}m_{k}^{t} \\
{[\mathrm{~kg}]}\end{array}$ & $\begin{array}{c}k_{k}{ }^{t} \\
{[\mathrm{kN} / \mathrm{m}]}\end{array}$ & $\begin{array}{c}\zeta_{k}{ }^{t} \\
{[-]}\end{array}$ \\
\hline 1 TMD & 2000 & 2.00 & 0.05 \\
2 TMDs & 1000 & 1.00 & 0.05 \\
4 TMDs & 500 & 0.50 & 0.05 \\
8 TMDs & 250 & 0.25 & 0.05 \\
20 TMDs & 100 & 0.10 & 0.05 \\
\hline
\end{tabular}

The calculation process involved using the aforementioned GA and SA optimization methods, and the objective functions based on $\mathrm{H}_{2}$ and $\mathrm{H} \infty$ norms in the form of $J 1$ and $J 2$. The frequency range when determining the objective function was $(0.0 \mathrm{rad} / \mathrm{s}: \pi \mathrm{rad} / \mathrm{s})$.

\subsection{Numerical optimization results - comparison of the GA and SA heuristic methods}

The efficiency of the GA and SA optimization was checked based on the example of 10 TMDs. Both heuristic methods do not guarantee finding an exact solution, which is the consequence of the very specificity of the methods. Figure 5 shows the optimal TMD parameter values obtained using both methods. Compared to SA, the GA method does not provide results convergent with the results presented in the work by Zuo and Nayfeh [19]. The obtained results are discussed in greater detail in Chapter 4. Only the SA optimization method was applied in the further part of the optimization calculations.

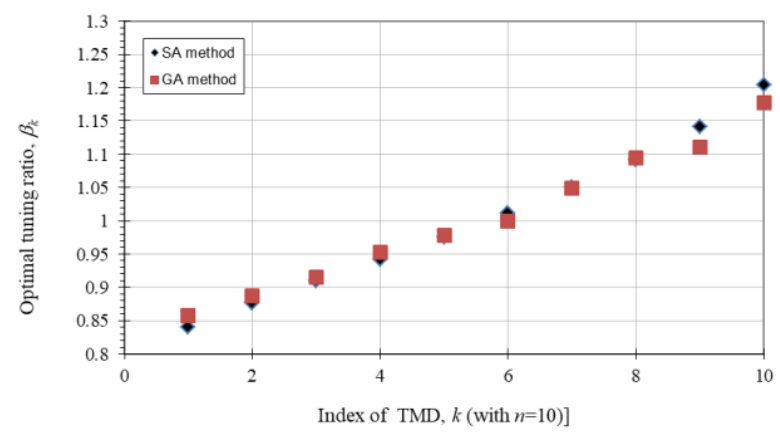

(a)

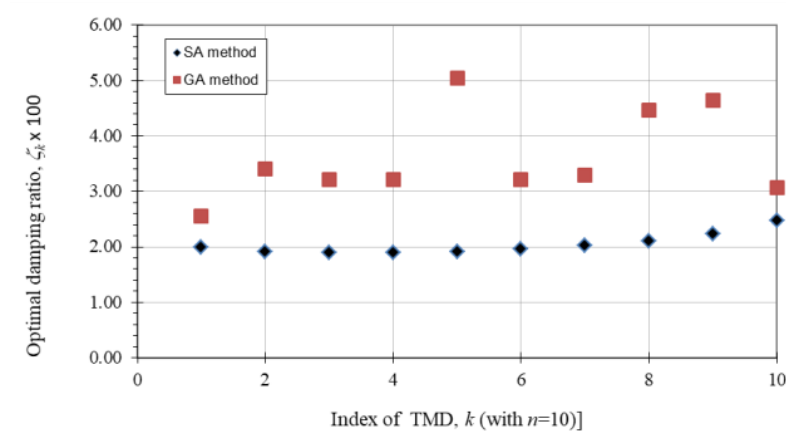

(b)

Figure 5. (a) Optimal tuning ratios $\beta_{k}$, (b) Optimal damping ratios $\zeta_{k}{ }^{t}$ of the individual TMD for $\mu=0.05$ and $\zeta_{1}=0$ for SA and GA method. 


\subsection{Numerical optimization results - SA method}

Optimal MTMD parameters, which are discussed below, were obtained as a result of computations. Table 2 shows the end values of the $J 1$ and $J 2$ objective functions, after the completion of the SA optimization process, using the $J 1$ and $J 2$ objective functions. In addition, it also specifies the percentage change of the objective function value, relative to a 1 TMD system. Computation accuracy was adopted at a level of $1 \mathrm{E}-10$.

Table 2. The value of the $J 1$ and $J 2$ functions after completed optimization with the SA method

\begin{tabular}{ccccc}
\hline TMD number & $\begin{array}{c}\text { J1 value } \\
{[\mathrm{s} / \mathrm{kg}]}\end{array}$ & $\begin{array}{c}\text { J1 change } \\
{[\%]}\end{array}$ & $\begin{array}{c}\text { J2 value } \\
{[\mathrm{m} / \mathrm{kN}]}\end{array}$ & $\begin{array}{c}\text { J2 change } \\
{[\%]}\end{array}$ \\
\hline SDOF & $7.5591 \mathrm{E}-03$ & & $2.5005 \mathrm{E}-01$ & \\
1 TMD & $6.3107 \mathrm{E}-03$ & $0.00 \%$ & $7.4579 \mathrm{E}-02$ & $0.00 \%$ \\
2 TMDs & $6.2650 \mathrm{E}-03$ & $-0.72 \%$ & $6.8248 \mathrm{E}-02$ & $-8.49 \%$ \\
4 TMDs & $6.2348 \mathrm{E}-03$ & $-1.20 \%$ & $6.4091 \mathrm{E}-02$ & $-14.06 \%$ \\
8 TMDs & $6.2158 \mathrm{E}-03$ & $-1.50 \%$ & $6.1620 \mathrm{E}-02$ & $-17.38 \%$ \\
20 TMDs & $6.2015 \mathrm{E}-03$ & $-1.73 \%$ & $6.0202 \mathrm{E}-02$ & $-19.28 \%$ \\
\hline
\end{tabular}

Table 3 and Table 4 show optimal MTMD parameters obtained with optimized J1 and J2 objective functions. A tabular presentation of the 20 TMDs was omitted due to the high number of the results. Results for these cases are shown as drawings in the next chapter. Figure 5a shows a graph of the transfer function module for an equivalent system $\left|\bar{H}_{41,41}\right|$, for a different number of TMDs and with applied J1 objective function, while Figure $5 b$ shows the same for the $J 2$ objective function.

Table 3. Optimal $k_{k}^{t}, \zeta_{k}^{t}, \beta_{k}$ values obtained for the $J 1$ objective function

\begin{tabular}{cccccc}
\hline MTMD & No. & $\begin{array}{c}m_{k}{ }^{t} \\
{[\mathrm{~kg}]}\end{array}$ & $\begin{array}{c}k_{k}{ }^{t} \\
{[\mathrm{kN} / \mathrm{m}]}\end{array}$ & $\begin{array}{c}\zeta_{k}{ }^{t} \\
{[-]}\end{array}$ & $\begin{array}{c}\beta_{k} \\
{[-]}\end{array}$ \\
\hline 1 TMD & 1 & 2000 & 1.960905 & 0.068462 & 0.990178 \\
\hline \multirow{2}{*}{ TMDs } & 1 & 1000 & 0.895498 & 0.041605 & 0.946307 \\
& 2 & 1000 & 1.096614 & 0.044381 & 1.047193 \\
\hline \multirow{3}{*}{ TMDs } & 1 & 500 & 0.419478 & 0.025349 & 0.915945 \\
& 2 & 500 & 0.470454 & 0.025186 & 0.970004 \\
& 3 & 500 & 0.526416 & 0.025998 & 1.026076 \\
& 4 & 500 & 0.595890 & 0.028082 & 1.091687 \\
\hline \multirow{3}{*}{ TMDs } & 1 & 250 & 0.200353 & 0.016536 & 0.895215 \\
& 2 & 250 & 0.214738 & 0.014990 & 0.926796 \\
& 3 & 250 & 0.228431 & 0.014593 & 0.955889 \\
& 4 & 250 & 0.242203 & 0.014669 & 0.984282 \\
& 5 & 250 & 0.256858 & 0.015086 & 1.013624 \\
& 6 & 250 & 0.273079 & 0.015356 & 1.045139 \\
& 7 & 250 & 0.291974 & 0.015877 & 1.080691 \\
& 8 & 250 & 0.316299 & 0.017789 & 1.124809 \\
\hline
\end{tabular}

Table 4. Optimal $k_{k}^{t}, \zeta_{k}^{t}, \beta_{k}$ values obtained for the $J 2$ objective function

\begin{tabular}{|c|c|c|c|c|c|}
\hline MTMD & No. & $\begin{array}{c}m_{k}^{t} \\
{[\mathrm{~kg}]}\end{array}$ & $\begin{array}{c}k_{k}^{t} \\
{[\mathrm{kN} / \mathrm{m}]}\end{array}$ & $\begin{array}{l}\zeta_{k}^{t} \\
{[-]}\end{array}$ & $\begin{array}{c}\beta_{k} \\
{[-]}\end{array}$ \\
\hline 1 TMD & 1 & 2000 & 1.905820 & 0.089169 & 0.976171 \\
\hline \multirow{2}{*}{2 TMDs } & 1 & 1000 & 0.878697 & 0.056278 & 0.937388 \\
\hline & 2 & 1000 & 1.046257 & 0.062921 & 1.022867 \\
\hline
\end{tabular}




\begin{tabular}{llllll}
\hline & 1 & 500 & 0.413107 & 0.036608 & 0.908964 \\
4 TMDs & 2 & 500 & 0.456409 & 0.038155 & 0.955415 \\
& 3 & 500 & 0.503223 & 0.039621 & 1.003218 \\
& 4 & 500 & 0.560626 & 0.041295 & 1.058892 \\
\hline \multirow{3}{*}{8 TMDs } & 1 & 250 & 0.198173 & 0.023339 & 0.890332 \\
& 2 & 250 & 0.210676 & 0.024023 & 0.917988 \\
& 4 & 250 & 0.222606 & 0.025988 & 0.943624 \\
& 5 & 250 & 0.233932 & 0.028452 & 0.967329 \\
& 6 & 250 & 0.245215 & 0.028899 & 0.990383 \\
& 7 & 250 & 0.258339 & 0.027081 & 1.016542 \\
& 8 & 250 & 0.274253 & 0.026689 & 1.047383 \\
\hline
\end{tabular}

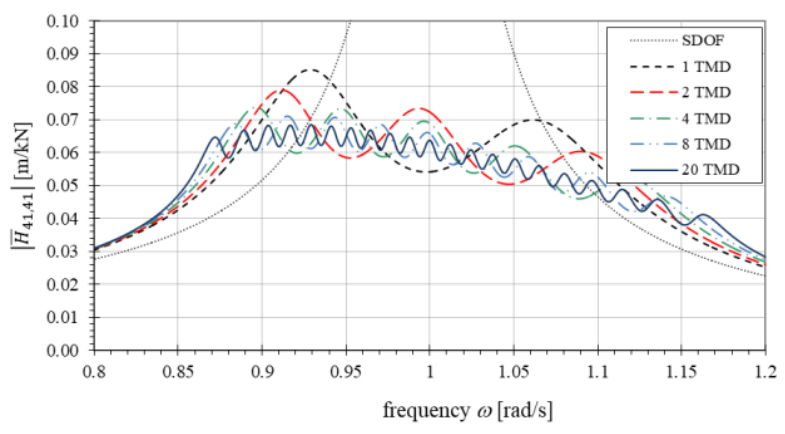

(a)

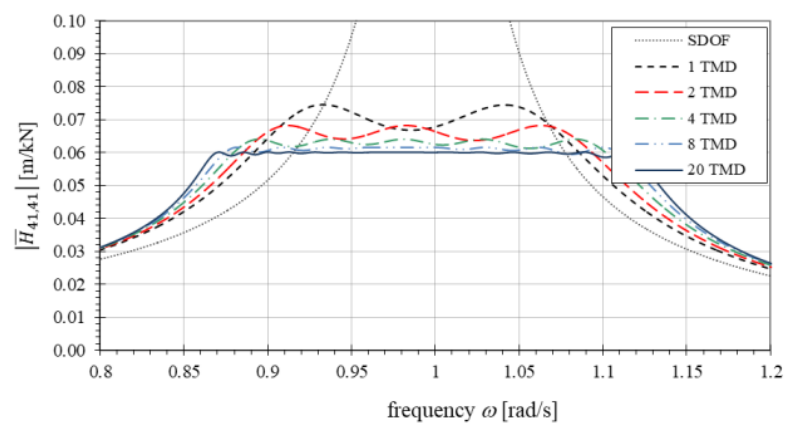

(b)

Figure 5. Module graph of a transfer function $\left|\bar{H}_{41,41}\right|$ for optimal parameters and a different number of TMDs; (a) for the J1 objective function; (b) for the J2 objective function.

\subsection{Impact of primary system mistuning}

The analysis also covered the impact by the changing mass of the equivalent structure $\bar{m}_{41,1}$, on the value of the equivalent structure transfer function $\left|\bar{H}_{41,41}\right|$ for a structure with attached MTMDs with optimal parameters, pre-determined based on the J1 and J2 objective function. A $10 \%$ reduction and increase in the equivalent structure mass was assumed, which results in the change of the natural circular frequencies to a value of $\omega_{1}^{-10 \%}=1.05409 \mathrm{rad} / \mathrm{s}$ and $\omega_{1}^{+10 \%}=0.95346 \mathrm{rad} / \mathrm{s}$, respectively. Figure 6a shows a graph of the transfer function module for an equivalent system $\left|\bar{H}_{41,41}\right|$, for a mass reduction of $10 \%$ and a different number of TMDs, and with applied J1 objective function, while Figure $6 \mathrm{~b}$ shows the same for the $J 2$ objective function. Figures $7 \mathrm{a}$ and $\mathrm{b}$ show analogous graphs but for a mass increase of $10 \%$ (designation $+10 \%$ and $-10 \%$ means an increase and reduction of the $\bar{m}_{41,1}$ equivalent mass, respectively).
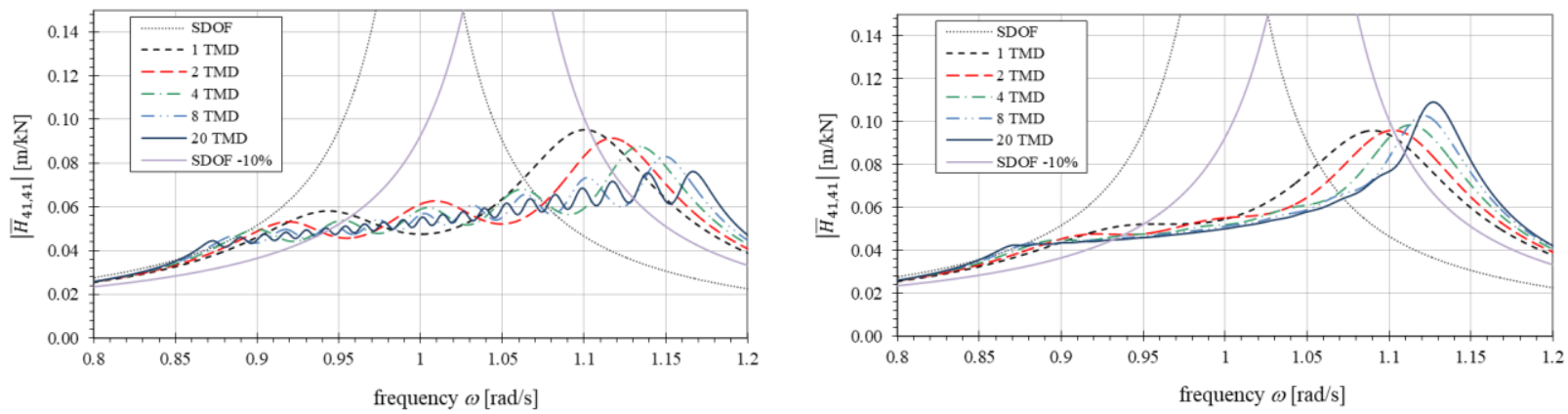
(a)

(b)

Figure 6. Module graph of a transfer function $\left|\bar{H}_{41,41}\right|$ with the mass of the primary system reduced by $10 \%$ for optimal parameters and a different number of TMDs; (a) for the J1 objective function; (b) for the $J 2$ objective function.

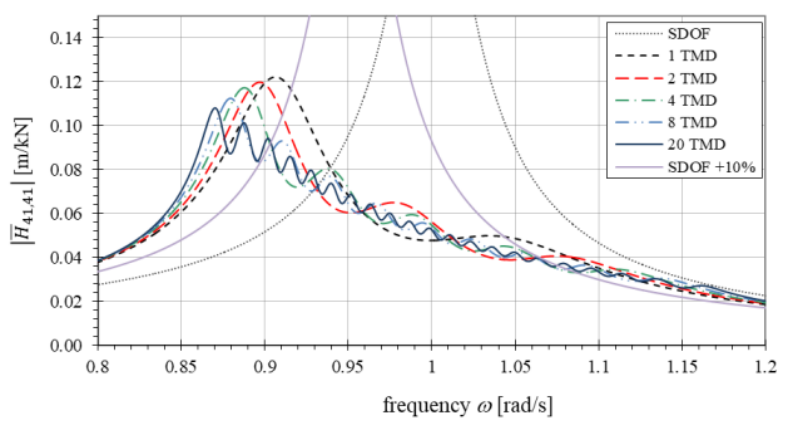

(a)

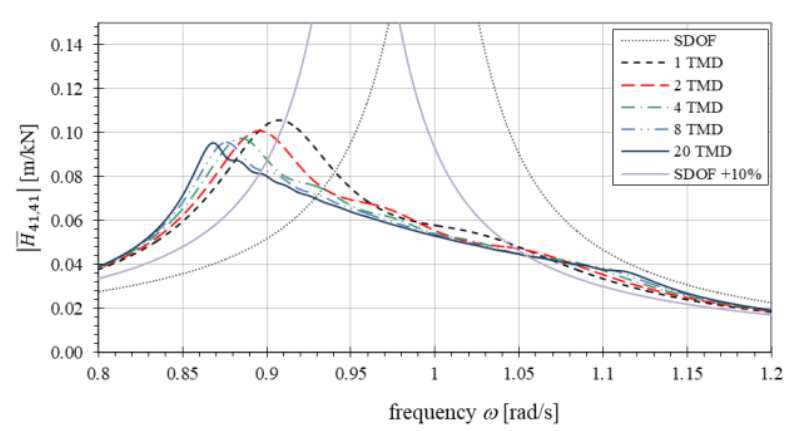

(b)

Figure 7. Module graph of a transfer function $\left|\bar{H}_{41,41}\right|$ with the mass of the primary system increased by $10 \%$ for optimal parameters and a different number of TMDs; (a) for the J1 objective function; (b) for the $J 2$ objective function.

\section{Discussion}

The analyses presented in chapter 3 were used as a base to compare the application of the SA optimization method and the $J 1$ and $J 2$ objective function with known analytical solutions in terms of a single TMD. Table 5 shows known formulas for the determination of the optimal parameters for TMD usually determined from a TDOF system, with stated values of the optimal parameters. The table also presents original results for $1 \mathrm{TMD}$, taking into account natural damping of the primary system, as well as without it. Figure 8 shows an equivalent system transfer function module graph for optimal TMD parameters. 
Table 5. Optimal parameters and J1 and J2 objective function values for $1 \mathrm{TMD}$, based on various authors (values for $\mu=0.02$ )

\begin{tabular}{|c|c|c|c|c|c|c|}
\hline & $\begin{array}{c}\text { Tuning } \\
\beta_{o p t}\end{array}$ & $\begin{array}{c}\text { Damping } \\
\zeta_{o p t}\end{array}$ & $\begin{array}{c}J 1 \text { value } \\
\zeta_{1}=0 \\
{[\mathrm{~s} / \mathrm{kg}]}\end{array}$ & $\begin{array}{c}J 2 \text { value } \\
\zeta_{1}=0 \\
{[\mathrm{~m} / \mathrm{kN}]}\end{array}$ & $\begin{array}{c}J 1 \text { value } \\
\zeta_{1}=0.02 \\
{[\mathrm{~s} / \mathrm{kg}]}\end{array}$ & $\begin{array}{c}J 2 \text { value } \\
\zeta_{1}=0.02 \\
{[\mathrm{~m} / \mathrm{kN}]}\end{array}$ \\
\hline Den Hartog & $\begin{array}{c}\frac{1}{1+\mu} \\
0.9803922\end{array}$ & $\begin{array}{l}\sqrt{\frac{3 \mu}{8(1+\mu)}} \\
0.0870388\end{array}$ & 7.051E-03 & $1.005 \mathrm{E}-01$ & 6.327E-03 & 7.676E-02 \\
\hline $\begin{array}{c}\text { Warburton } \\
\text { (1981) }\end{array}$ & $\begin{array}{c}\frac{1}{1+\mu} \sqrt{\frac{2+\mu}{2}} \\
0.9852819\end{array}$ & $\begin{array}{c}\sqrt{\frac{\mu(4+3 \mu)}{8(1+\mu)(2+\mu)}} \\
0.0701871\end{array}$ & 7.019E-03 & $1.091 \mathrm{E}-01$ & 6.312E-03 & $8.176 \mathrm{E}-02$ \\
\hline $\begin{array}{c}\text { Warburton } \\
\text { (1982) }\end{array}$ & $\begin{array}{l}\frac{1}{1+\mu} \sqrt{\frac{2-\mu}{2}} \\
0.9754779\end{array}$ & $\begin{array}{c}\sqrt{\frac{3 \mu}{8(1+\mu)(1-\mu / 2)}} \\
0.0861813\end{array}$ & 7.060E-03 & 1.057E-01 & $6.331 \mathrm{E}-03$ & 7.497E-02 \\
\hline Ren & $\begin{array}{c}\sqrt{\frac{1}{1-\mu}} \\
1.0101525\end{array}$ & $\begin{array}{c}\sqrt{\frac{3 \mu}{8(1-\mu / 2)}} \\
0.0870388\end{array}$ & $7.089 \mathrm{E}-03$ & $1.366 \mathrm{E}-01$ & - & - \\
\hline $\begin{array}{c}\text { Nishihara and } \\
\text { Matsuhisa }\end{array}$ & $\begin{array}{c}\frac{1}{1+\mu}\left(1-\zeta_{1} \sqrt{\frac{\mu}{1+\mu-\zeta_{1}^{2}}}\right) \\
0.9776460\end{array}$ & $\begin{array}{c}\frac{\zeta_{1}+\sqrt{\mu\left(1+\mu-\zeta_{1}^{2}\right)}}{1+\mu} \\
0.1596084\end{array}$ & - & - & $6.484 \mathrm{E}-03$ & 9.865E-02 \\
\hline $\begin{array}{c}\text { Proposed J1 } \\
\zeta_{1}=0\end{array}$ & $\begin{array}{c}- \\
0.9890635\end{array}$ & $\begin{array}{c}- \\
0.0682140\end{array}$ & 7.017E-03 & $1.148 \mathrm{E}-02$ & - & - \\
\hline $\begin{array}{c}\text { Proposed } J 2 \\
\zeta_{1}=0\end{array}$ & $\begin{array}{c}- \\
0.9803647\end{array}$ & $\begin{array}{c}- \\
0.0853719\end{array}$ & 7.050E-03 & $1.006 \mathrm{E}-02$ & - & - \\
\hline $\begin{array}{c}\text { Proposed J1 } \\
\zeta_{1}=0.02\end{array}$ & $\begin{array}{c}- \\
0.9901779\end{array}$ & $\begin{array}{c}- \\
0.0684623\end{array}$ & - & - & 6.311E-03 & 8.521E-02 \\
\hline $\begin{array}{c}\text { Proposed J2 } \\
\zeta_{1}=0.02\end{array}$ & $\begin{array}{c}- \\
0.9761710\end{array}$ & $\begin{array}{c}- \\
0.0891687\end{array}$ & - & - & 6.334E-03 & 7.458E-02 \\
\hline
\end{tabular}

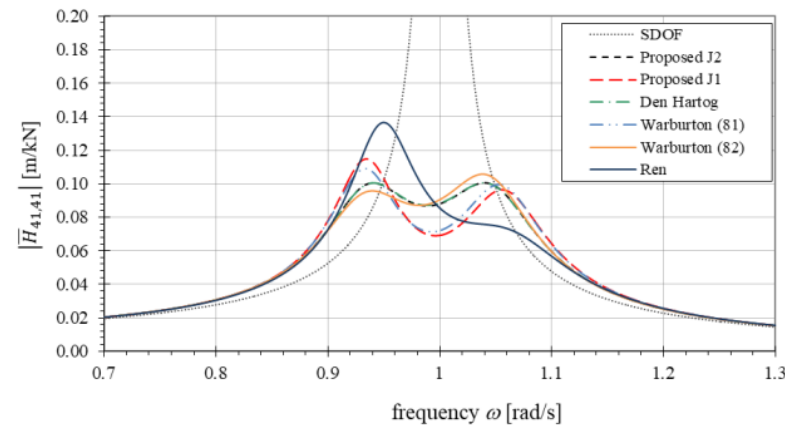

(a)

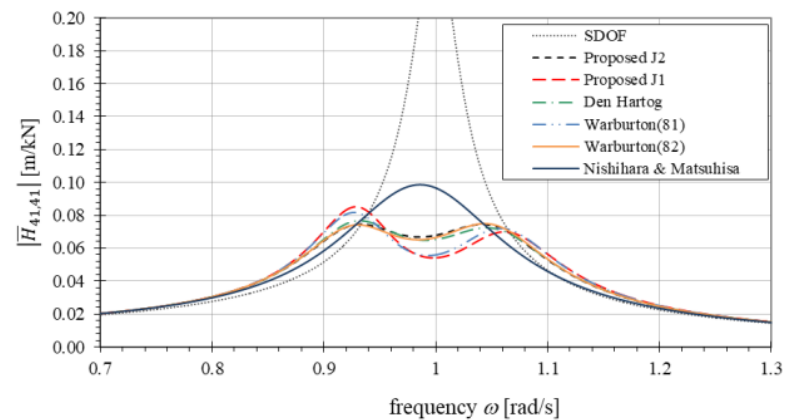

(b)

Figure 8. The module graph for the transfer function $\left|\bar{H}_{41,41}\right|$ (value corresponds to the transfer function $\left|H_{1,1}\right|$ of a TDOF system) based on various studies; (a) case $\zeta_{1}=0 ;(\mathbf{b})$ case $\zeta_{1}=0.02$.

When analyzing the obtained solution (see Table 6 and Figure 8a), in the case of no damping of the primary system $\zeta_{1}=0$ and in comparison with the Den Hartog solution [1], which has been known for years, on can state very good conformity of the optimal tuning parameters $\beta_{1}$ and the 
TMD damping factor $\zeta_{1}{ }^{t}$. Whereas optimal TMD parameters proposed by Warburton [2], upon a harmonic excitation are close to the parameters obtained through calculations of the $J 2$ objective function optimization. The solution proposed by Ren [30] suits a different kind of TMDs, where the damping element is not connected with the primary mass but with the substrate (other motion equations), hence, it is impossible to directly apply the optimal parameter formulas proposed in this study as parameters for a standard TDOF system. Furthermore, please note that optimal TMD parameters based on the $\mathrm{H}_{2}$ norm (white noise input), i.e., determined from the $\mathrm{J} 1$ objective function, provide similar TMD efficiency as suggested by Warburton in [3].

In the case of an analysis covering a structure including natural damping of the primary system, we can observe that transfer function module graphs, developed based on optimal Den Hartog and Warburton parameters, are no longer consistent with the graphs based on the parameters obtained through the $\mathrm{H}_{2}$ and $\mathrm{H}_{\infty}$ methods, that is, the $J 1$ and $J 2$ objective functions. This, of course, stems from the fact that these formulas do not include natural damping $\zeta_{1}$. Whereas, when comparing to the Matsuhis solution [31], where the optimal parameters were obtained based on the stability criterion, taking into account $\zeta_{1}$, it should be concluded that his solution was optimal neither for harmonic excitations, as well as white noise input.

In [19], Zuo and Nayfeh conducted a free analysis of the $\beta_{k}, \zeta_{k}^{t}$ parameters, with a constant value of $m_{k}{ }^{t}$, for 10 and 100 TMDs, with not damping of the primary system $\zeta_{1}=0$ and a $\mu=0.05$ mass ratio. They applied optimization based on the $\mathrm{H}_{2}$ norm and used state space equations for an SDOF system with attached MTMDs. The work also presents tuning increment graphs for individual TMDs in the form of $\beta_{k}-\beta_{k-1}$. The authors of that article also conducted such simulations for $n=10$ (number of TMD), $\zeta_{1}=0$ and $\mu=0.05$. Figure 9 shows tuning $\beta_{k}$ and damping ratio $\zeta_{k}$ distribution based on the number of TMDs, while Figure 10 shows tuning increments and tuning factor for individual TMDs in the form of $\beta_{k}-\beta_{k-1}$ and $\zeta_{k}-\zeta_{k-1}$. The obtained $\beta_{k}, \zeta_{k}^{t}$ and $\beta_{k}-\beta_{k-1}$ parameters exhibited very good conformity with the results of Zuo and Nayfeh [19].
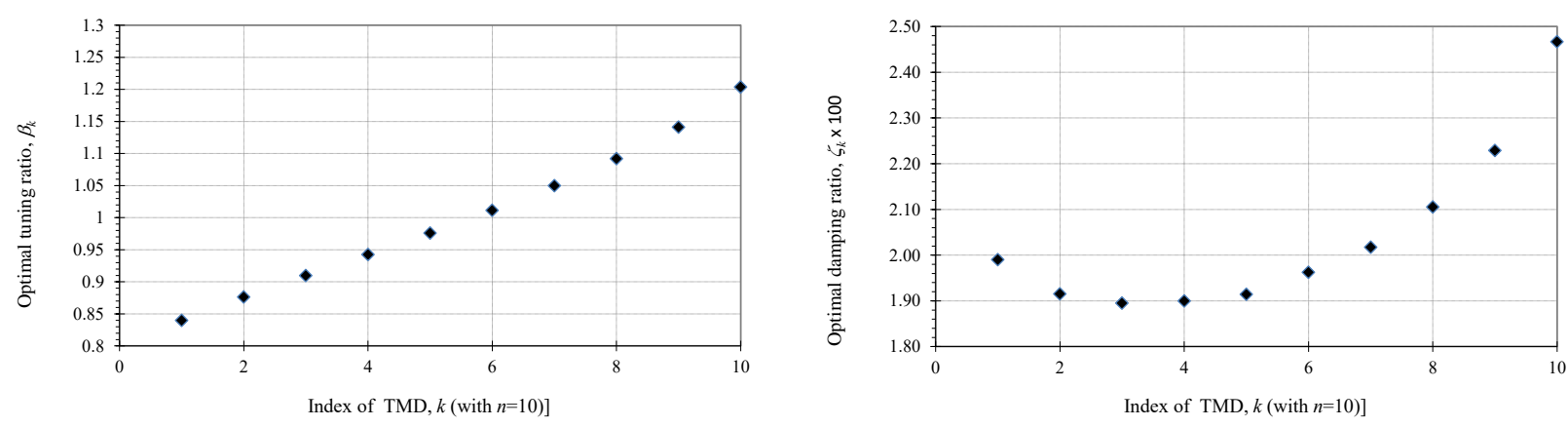

Figure 9. Optimal tuning ratios $\beta_{k}$ and damping ratios $\zeta_{k}^{t}$ of the individual TMD for $\mu=0.05$ and $\zeta_{1}=0$ obtained on the basis of $J 1$.
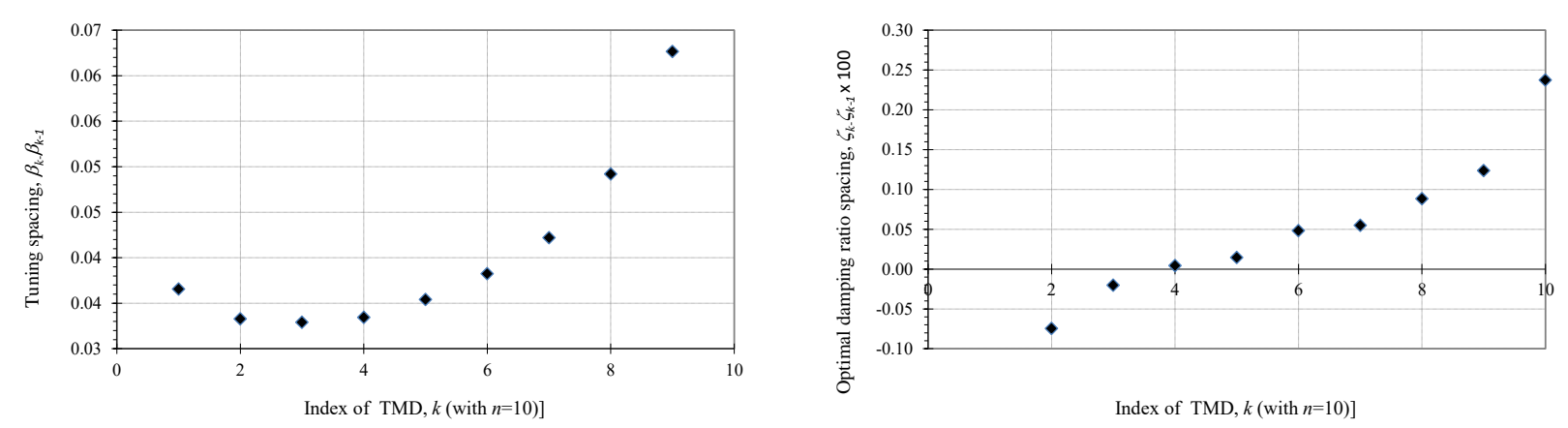
Figure 10. Optimal tuning spacings $\beta_{k}-\beta_{k-1}$ and damping ratios spacings $\zeta_{k}-\zeta_{k-1}$ of the individual TMD for $\mu=0.05$ and $\zeta_{1}=0$ obtained on the basis of $J 1$.

In the course of analyzing the results shown in chapter 3, which concern the optimization of MTMDs located at the tip of a $h=160 \mathrm{~m}$ high chimney, higher effectiveness of MTMDs relative to a single TMD can be confirmed (see Table 2 and Figure 5). This is particularly clear to optimal parameters determined with the $J 2$ objective function, where the location of the flat section of graph $\left|\bar{H}_{41,41}\right|$ in the area of $\omega_{1}$ can be seen increasingly lower (Figure 5b). Another issue that needs to be stressed are the graphs $\left|\bar{H}_{41,41}\right|$ (Figure 5a) for TMD tuning in the form of white noise $\left(\mathrm{H}_{2}\right.$ optimization of the $J 1$ function) exhibit a broader damper impact range on the side of higher input frequencies.

Furthermore, Figure 11 shows a function variability graph depending on the number of TMDs. Of course, higher MTMD efficiency relative to TMDs can be observed for both objective functions, J1 and J2, applied within the numerical optimization. Furthermore, Figure 11 shows the values of individual objective functions for the optimal parameters obtained using both optimization methods, namely, $\mathrm{H}_{2}$ (J1 function) and $\mathrm{H}_{\infty}$ (J2 function). It can be seen (see Figure 11a) that in the case of random excitation, which corresponds to the value of the J1 function and TMDs tuned to harmonic excitation (optical parameters based on the optimization of function $J 2$ ), we get a lower MTMD efficiency. Figure $11 b$ indicates an inverse relationship.

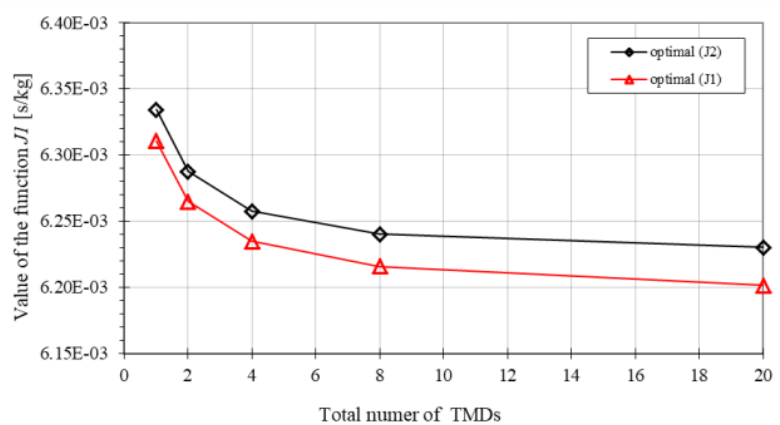

(a)

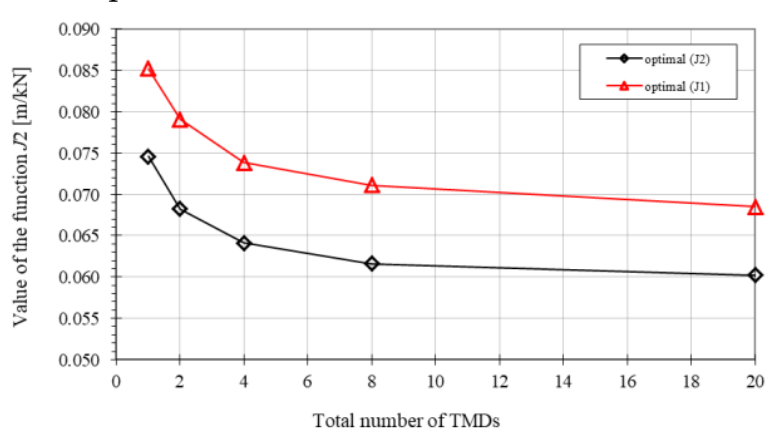

(b)

Figure 11. Objective function values based on the optimal parameters obtained from $J 1$ and $J 2$, depending on the number of TMDs, (a) value of $J 1,(\mathbf{b})$ value of $J 2$

When analyzing the results concerning the primary system mass change (see Figure 6, 7), for an approximately $5 \%$ difference in the value of $\omega_{1}$ ("structural mistuning"), MTMDs are more effective than a single TMD. A lower effectiveness with a higher number of TMDs tuned to harmonic excitations can be observed only in the case of TMDs tuned based on J2 optimization and reducing the primary system mass (increase in frequency $\omega_{1}^{-10 \%}=1.05409 \mathrm{rad} / \mathrm{s}$. In the event of such a primary system mistuning, better effectiveness is exhibited by MTMDs tuned based on J1, which results from the aforementioned broadband excitation on the side of frequencies higher than $\omega_{1}$. It can also be concluded that a reduction in the primary system mass has a more adverse influence on the structural response and MTMD operation than mass increase. These relationships are shown in Figure 12. It can also be assumed that with a low difference (below 5\%) between the calculated natural frequencies of the structure and the ones determined for a real structure, it is advisable to use MTMDs instead of a single TMD. The aforementioned analyses confirm the general conclusions included in the works by Zuo and Nayfeh [19], as well as $\mathrm{Li}$ and $\mathrm{Ni}$ [20] concerning the higher effectiveness of MTMDs relative to TMDs. 


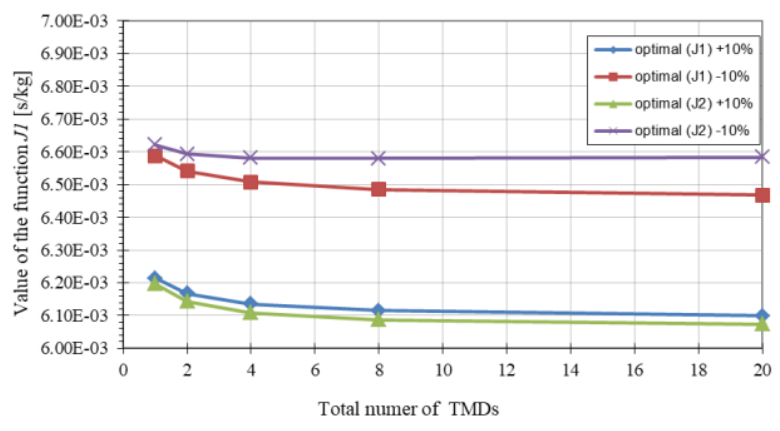

(a)

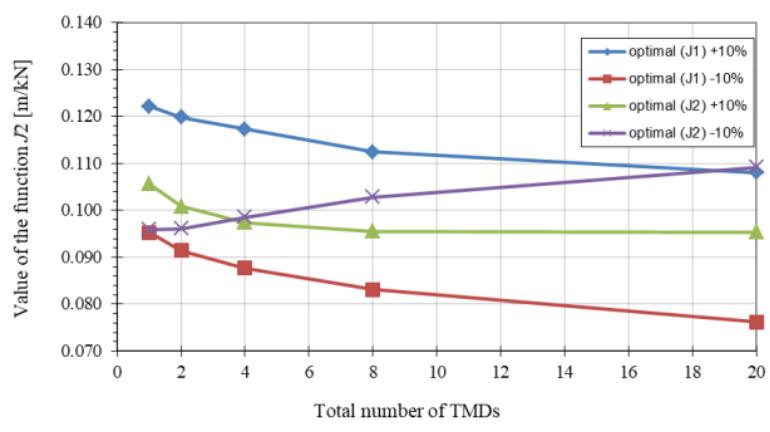

(b)

Figure 12. Objective function value, in the case of primary system mistuning ( $10 \%$ increase and drop of mass) based on the optimal parameters obtained from $J 1$ and $J 2$, depending on the number of TMDs, (a) value of $J 1$, (b) value of $J 2$

Additionally, for analysis purposes, it was decided to present the results of optimal MTMD parameters in the form of optimal tuning ratio $\beta_{k}$ and optimal damping ratio $\zeta_{k}^{t}$ graphs depending on the number of TMDs. The tuning and damping factor increments for individual TMDs in the form of $\beta_{k}-\beta_{k-1}$ and $\zeta_{k}^{t}-\zeta_{k-1}^{t}$ were also presented. A thorough review of the graphs (see Figures 12, 13 and 14) presented for cases with 4, 8 and 20 TMDs, conclusions can be drawn regarding the application of the $J 1$ objective function based on the $\mathrm{H}_{2}$ norm, and the $J 2$ function based on the $\mathrm{H}_{\infty}$ norm. As far as the tuning distribution is concerned, we can observe increased factor $\beta_{k}$ for TMDs tuned above and below the $\omega_{1}$ frequency. The area of TMDs tuned to values beyond $\omega_{1}$ exhibits a significantly higher growth of the value $\beta_{k}$, especially for the parameters obtained from the $J 1$ objective function, which is particularly well illustrated by the graphs $\beta_{k}-\beta_{k-1}$. If we consider the optimal tuning ratio graphs for individual $\zeta_{k}^{t}$, we can observe a derivative characteristic, but only for the J1 function, although the increments $\zeta_{k}^{t}-\zeta_{k-1}^{t}$ no longer show a similar dependency as $\beta_{k}-\beta_{k-1}$. On the other hand, the optimal parameters $\zeta_{k}{ }^{t}$ obtained based on the $J 2$ function look completely different. In this case, the graph shows a significant drop in the $\zeta_{k}^{t}$ values for "central" TMDs tuned on $\omega_{1}$. 

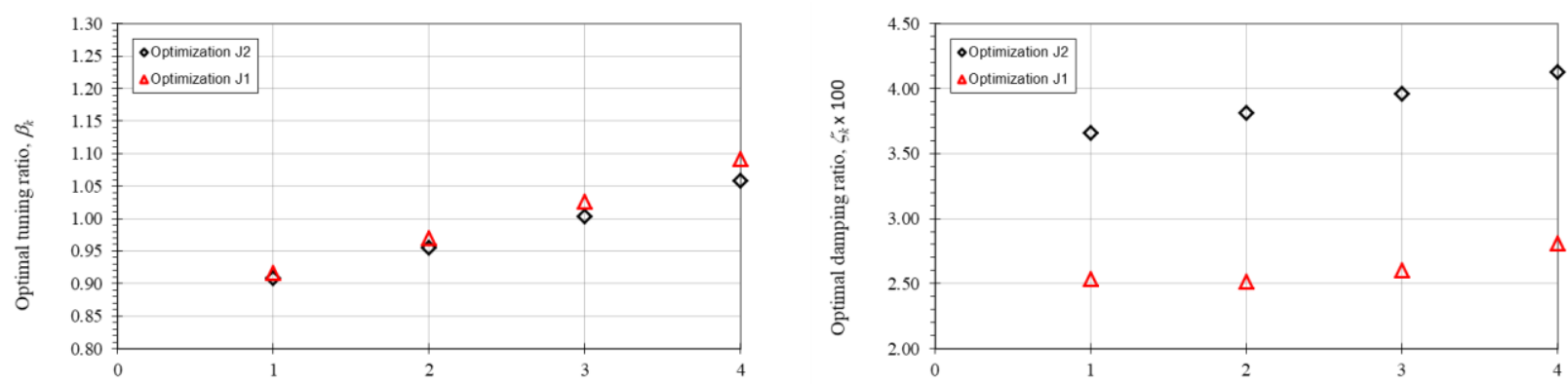

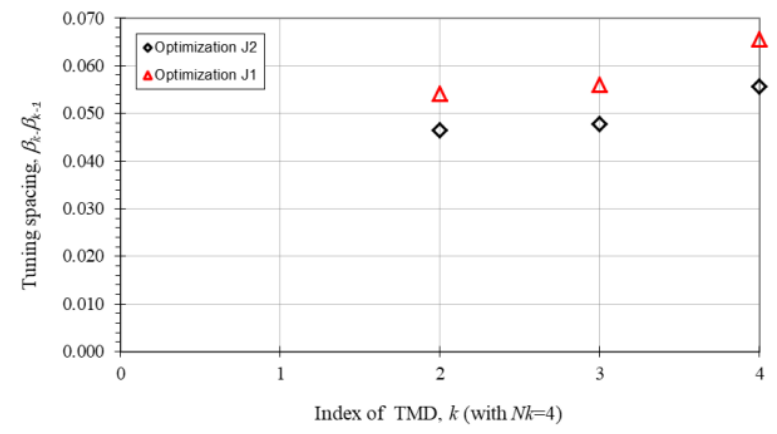

(a)

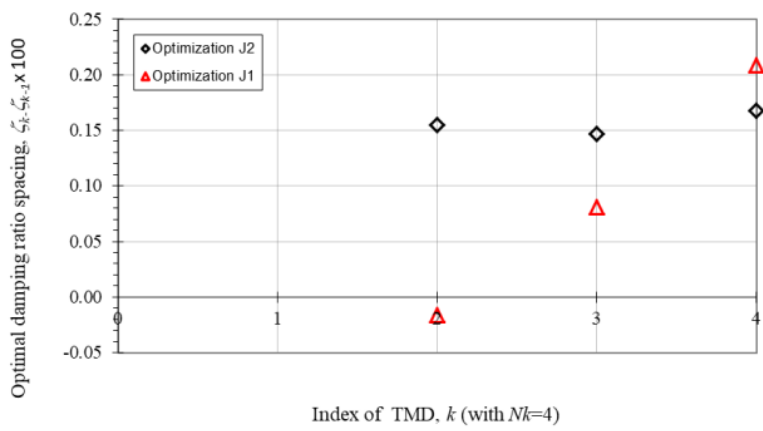

(b)

Figure 12. (a) Optimal tuning ratios $\beta_{k}$ and optimal tuning spacings $\beta_{k}-\beta_{k-1}$ (b) Optimal damping ratios $\zeta_{k}^{t}$ and damping ratios spacings $\zeta_{k}-\zeta_{k-1}$ of the individual TMD for $N_{k}=4, \mu=0.02$ and $\zeta_{1}=0.02$.
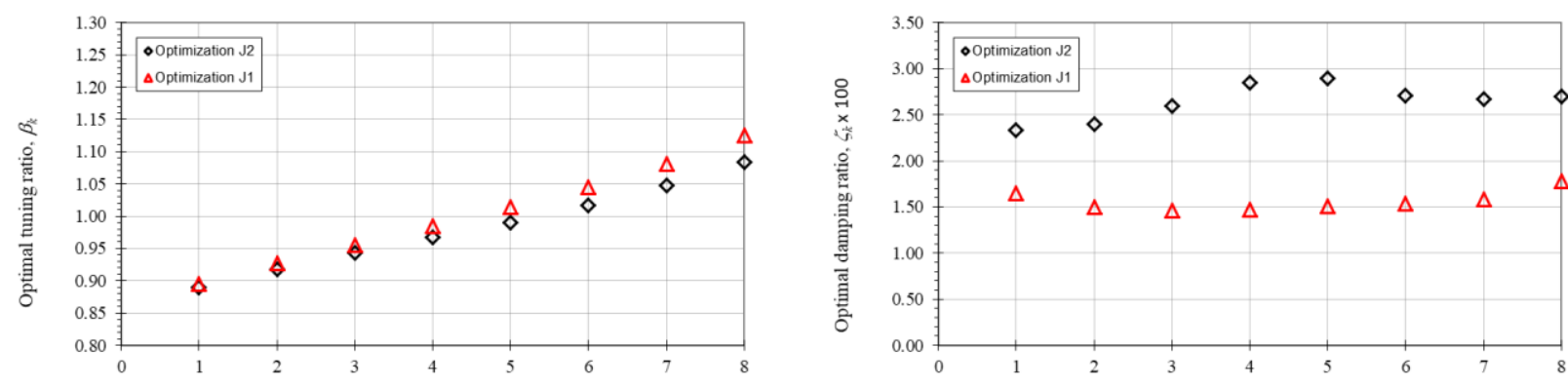

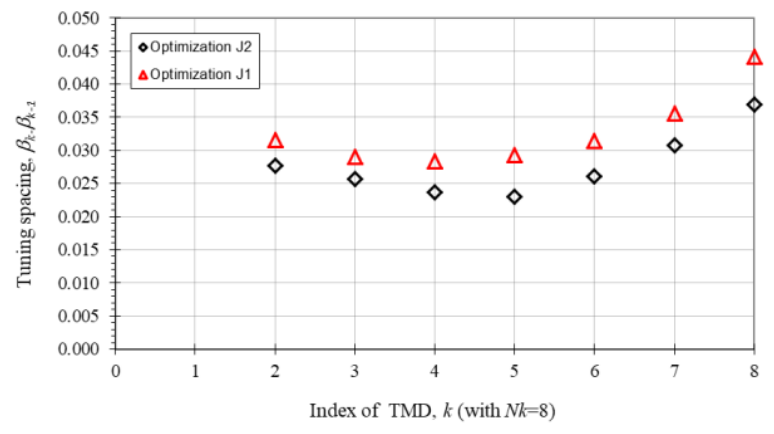

(a)

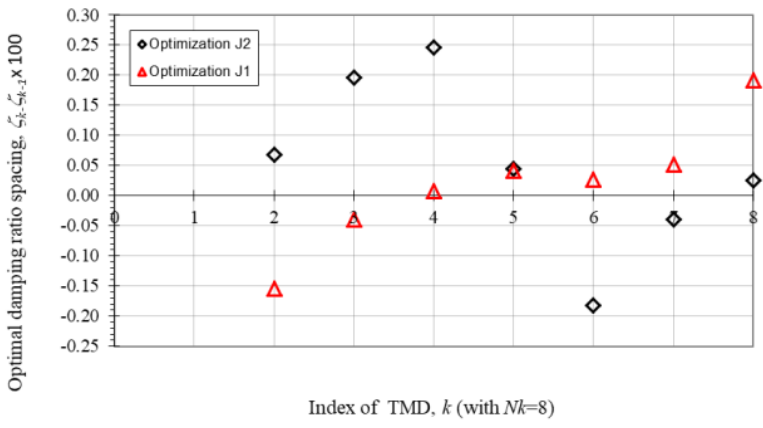

(b)

Figure 13. (a) Optimal tuning ratios $\beta_{k}$ and optimal tuning spacings $\beta_{k}-\beta_{k-1}$ (b) Optimal damping ratios $\zeta_{k}^{t}$ and damping ratios spacings $\zeta_{k}-\zeta_{k-1}$ of the individual TMD for $N_{k}=8, \mu=0.02$ and $\zeta_{1}=0.02$. 

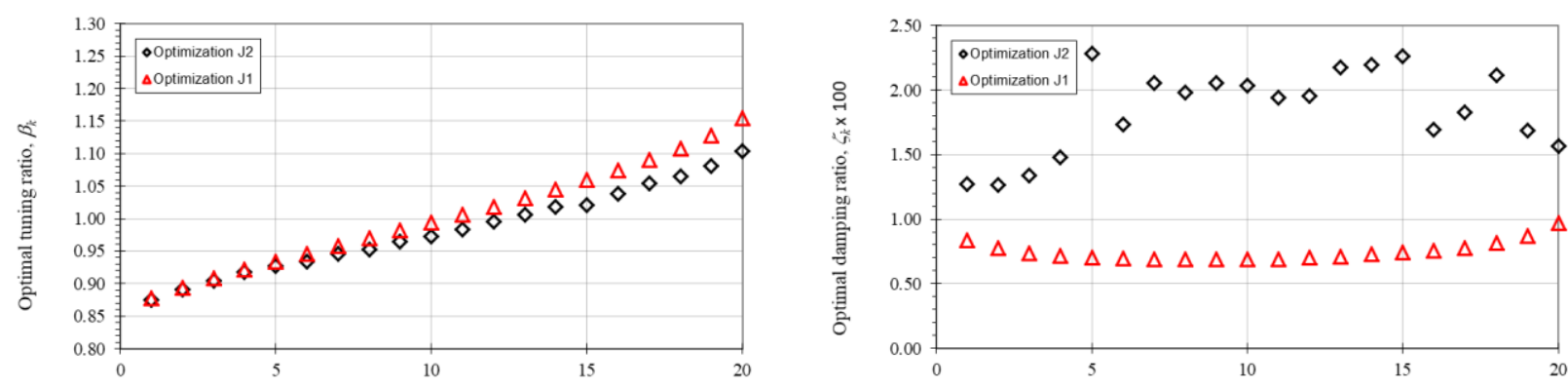

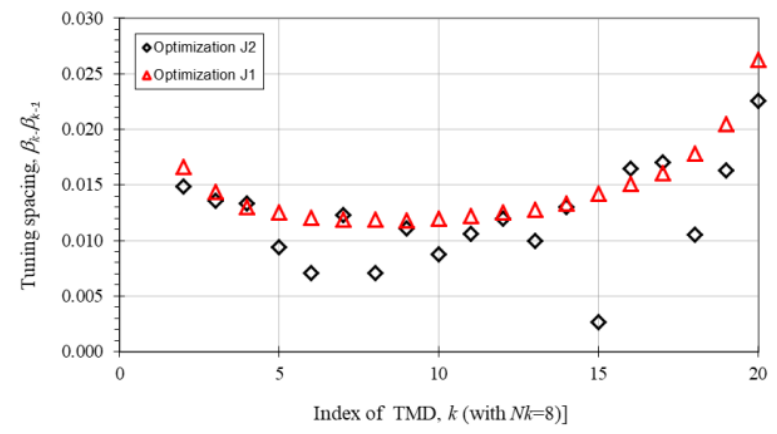

(a)

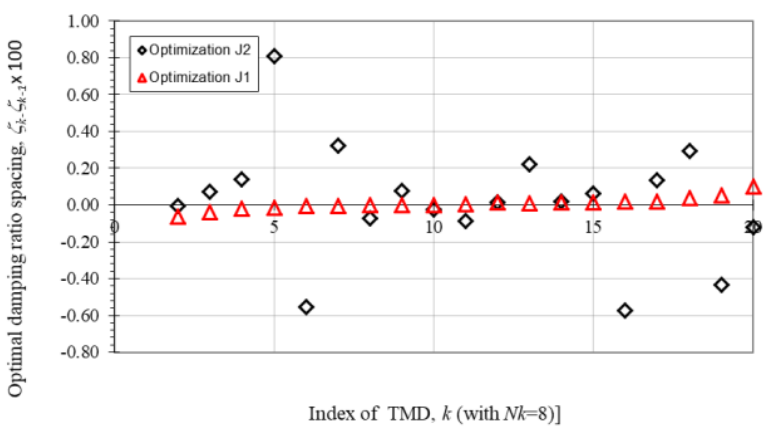

(b)

Figure 14. (a) Optimal tuning ratios $\beta_{k}$ and optimal tuning spacings $\beta_{k}-\beta_{k-1}$ (b) Optimal damping ratios $\zeta_{k}{ }^{t}$ and damping ratios spacings $\zeta_{k}-\zeta_{k-1}$ of the individual TMD for $N_{k}=20, \mu=0.02$ and $\zeta_{1}=0.02$.

\section{Conclusions}

The adopted new method for developing motion equations enables adding single TMDs or MTMDs to completely different degrees of freedom of the primary system. The system of equations allows for easy MTMD tuning for complex vibrations modes, with MTMDs located in local maxima of these vibrations modes, while still analyzing the SDOF system with attached MTMDs. Locating MTMDs at the tip of the chimney provided a possibility to compare the results with studies on MTMD tuning. The next stage of the research will involve the parameter optimization in MTMDs unevenly distributed within the primary system, but still analyzed as an SDOF equivalent system.

Based on conducted analysis, it was observed that the $k_{k}{ }^{t}, \zeta_{k}{ }^{t}$ free optimization was of completely different nature in the case of optimizations based on the $\mathrm{H}_{2}$ and $\mathrm{H}_{\infty}$ norms, especially for the $\zeta_{k}^{t}$ parameters, where the $\zeta_{k}^{t}-\zeta_{k-1}^{t}$ increments show totally different forms for both J1 and J2 modulus of objective function.

Author Contributions: Conceptualization, P.W.; R.G., Formal analysis, P.W.; Investigation, P.W.; Methodology, P.W.; R.G., Resources, P.W.; Software, P.W.; Supervision, P.W.; Validation, R.G.; Visualization, R.G.; Writing-original draft, P.W.; Writing-review and editing, R.G. All authors have read and agreed to the published version of the manuscript.

Funding: This research was funded by the Science Financing Subsidy Lublin University of Technology (FN16/ILT/2020).

Conflicts of Interest: The authors declare no conflict of interest.

\section{References}

1. Den Hartog, J.P.D. Mechanical Vibration; McGraw-Hill: New York, 1956;

2. Warburton, G.B. Optimum absorber parameters for various combinations of response and 
excitation parameters. Earthq. Eng. Struct. Dyn. 1982, 10, 381-401, doi:10.1002/eqe.4290100304.

3. Warburton, G.B. Optimum absorber parameters for minimizing vibration response. Earthq. Eng. Struct. Dyn. 1981, 9, 251-262, doi:10.1002/eqe.4290090306.

4. Bakre, S. V.; Jangid, R.S. Optimum parameters of tuned mass damper for damped main system. Struct. Control Heal. Monit. 2007, 14, 448-470, doi:10.1002/stc.166.

5. Bakre, S. V.; Jangid, R.S. Optimum multiple tuned mass dampers for base-excited damped main system. Int. J. Struct. Stab. Dyn. 2004, 4, 527-542, doi:10.1142/s0219455404001367.

6. Krenk, S. Frequency analysis of the tuned mass damper. J. Appl. Mech. Trans. ASME 2005, 72, 936-942, doi:10.1115/1.2062867.

7. Batou, A.; Adhikari, S. Optimal parameters of viscoelastic tuned-mass dampers. J. Sound Vib. 2019, 445, 17-28, doi:10.1016/J.JSV.2019.01.010.

8. Marian, L.; Giaralis, A. The tuned mass-damper-inerter for harmonic vibrations suppression, attached mass reduction, and energy harvesting. Smart Struct. Syst. 2017, 19, 665-678, doi:10.12989/sss.2017.19.6.665.

9. Sentyakov, K.; Peterka, J.; Smirnov, V.; Bozek, P.; Sviatskii, V. Modeling of boring mandrel working process with vibration damper. Materials (Basel). 2020, 13, 1931, doi:10.3390/MA13081931.

10. Bergman, L.A.; McFarland, D.M.; Hall, J.K.; Johnson, E.A.; Kareem, A. Optimal distribution of tuned mass dampers in wind-sensitive structures. In Proceedings of the Proceedings of ICOSSAR '89, the 5th International Conference on Structural Safety and Reliability, Part I San Francisco, CA, USA; Publ by ASCE, 1989; pp. 95-102.

11. Kareem, A.; Kline, S. Performance of Multiple Mass Dampers under Random Loading. J. Struct. Eng. 1995, 121, 348-361, doi:10.1061/(asce)0733-9445(1995)121:2(348).

12. Igusa, T.; Xu, K. Vibration Control Using Multiple Tuned Mass Dampers. J. Sound Vib. 1994, 175, 491-503, doi:10.1006/jsvi.1994.1341.

13. Joshi, A.S.; Jangid, R.S. Optimum parameters of multiple tuned mass dampers for base-excited damped systems. J. Sound Vib. 1997, 202, 657-667, doi:10.1006/jsvi.1996.0859.

14. Jangid, R.S. Optimum Multiple Tuned Mass Dampers for base-excited undamped system. Earthq. Eng. Struct. Dyn. 1999, 28, 1041-1049, doi:10.1002/(SICI)1096-9845(199909)28:9<1041::AID-EQE853>3.0.CO;2-E.

15. Yamaguchi, H.; Harnpornchai, N. Fundamental characteristics of Multiple Tuned Mass Dampers for suppressing harmonically forced oscillations. Earthq. Eng. Struct. Dyn. 1993, 22, 51-62, doi:10.1002/eqe.4290220105.

16. Zuo, L.; Nayfeh, S.A. The two-degree-of-freedom tuned-mass damper for suppression of single-mode vibration under random and harmonic excitation. J. Vib. Acoust. Trans. ASME 2006, 128, 56-65, doi:10.1115/1.2128639.

17. Zhao, G.; Raze, G.; Paknejad, A.; Deraemaeker, A.; Kerschen, G.; Collette, C. Active tuned inerter-damper for smart structures and its $\mathscr{H} \infty$ optimisation. Mech. Syst. Signal Process. 2019, 129, 470-478, doi:10.1016/j.ymssp.2019.04.044.

18. Aggumus, H.; Guclu, R. Robust Hळ control of STMDs used in structural systems by hardware in the loop simulation method. Actuators 2020, 9, 55, doi:10.3390/ACT9030055.

19. Zuo, L.; Nayfeh, S.A. Optimization of the individual stiffness and damping parameters in multiple-tuned-mass-damper systems. J. Vib. Acoust. Trans. ASME 2005, 127, 77-83, doi:10.1115/1.1855929. 
20. Li, H.N.; Ni, X.L. Optimization of non-uniformly distributed multiple tuned mass damper. J. Sound Vib. 2007, doi:10.1016/j.jsv.2007.07.014.

21. Zuo, L.; Nayfeh, S.A. Minimax optimization of multi-degree-of-freedom tuned-mass dampers. J. Sound Vib. 2004, 272, 893-908, doi:10.1016/S0022-460X(03)00500-5.

22. Ok, S.Y.; Song, J.; Park, K.S. Optimal design of hysteretic dampers connecting adjacent structures using multi-objective genetic algorithm and stochastic linearization method. Eng. Struct. 2008, 30, 1240-1249, doi:10.1016/j.engstruct.2007.07.019.

23. Leung, A.Y.T.; Zhang, H. Particle swarm optimization of tuned mass dampers. Eng. Struct. 2009, 31, 715-728, doi:10.1016/j.engstruct.2008.11.017.

24. Zhang, R.; Zhang, Y.; Zheng, Z.; Mo, L.; Wu, C. Parametrical optimization of particle dampers based on particle swarm algorithm. Appl. Acoust. 2020, 160, 107083, doi:10.1016/j.apacoust.2019.107083.

25. Aydin, E.; Öztürk, B.; Dutkiewicz, M. Analysis of efficiency of passive dampers in multistorey buildings. J. Sound Vib. 2019, 439, 17-28, doi:10.1016/j.jsv.2018.09.031.

26. Kim, S.Y.; Lee, C.H. Optimum design of linear multiple tuned mass dampers subjected to white-noise base acceleration considering practical configurations. Eng. Struct. 2018, doi:10.1016/j.engstruct.2018.06.002.

27. Stanikzai, M.H.; Elias, S.; Matsagar, V.A.; Jain, A.K. Seismic response control of base-isolated buildings using tuned mass damper. Aust. J. Struct. Eng. 2019, doi:10.1080/13287982.2019.1635307.

28. Yin, X.; Song, G.; Liu, Y. Vibration Suppression of Wind/Traffic/Bridge Coupled System Using Multiple Pounding Tuned Mass Dampers (MPTMD). Sensors 2019, 19, 1133, doi:10.3390/s19051133.

29. Bendat, J.S.; Piersol, A.G. Random Data; Wiley Series in Probability and Statistics; John Wiley \& Sons, Inc.: Hoboken, NJ, USA, 2010; ISBN 9781118032428.

30. Ren, M.Z. A variant design of the dynamic vibration absorber [3]. J. Sound Vib. 2001, 245, 762770 .

31. Nishihara, O.; Matsuhisa, H. Design of a dynamic vibration absorber for minimization of maximum amplitude magnification factor (Derivation of algebraic exact solution). Nippon Kikai Gakkai Ronbunshu, C Hen/Transactions Japan Soc. Mech. Eng. Part C 1997, 63, 3438-3445, doi:10.1299/kikaic.63.3438. 